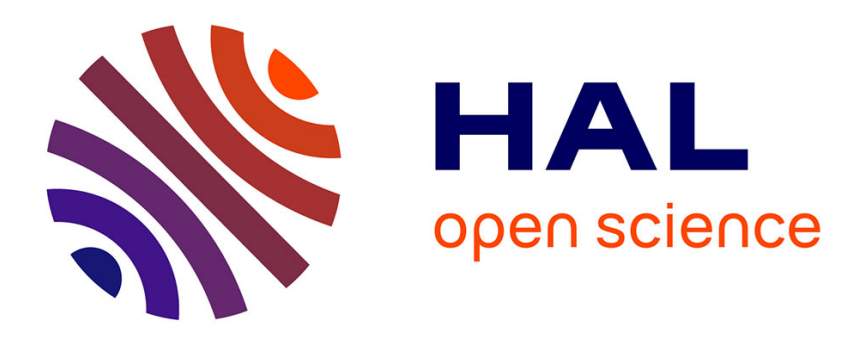

\title{
Light-dependent orientation responses in animals can be explained by a model of compass cue integration
}

\author{
Kenneth Kragh Jensen
}

\section{To cite this version:}

Kenneth Kragh Jensen. Light-dependent orientation responses in animals can be explained by a model of compass cue integration. Journal of Theoretical Biology, 2009, 262 (1), pp.129. 10.1016/j.jtbi.2009.09.005 . hal-00554644

\section{HAL Id: hal-00554644 \\ https://hal.science/hal-00554644}

Submitted on 11 Jan 2011

HAL is a multi-disciplinary open access archive for the deposit and dissemination of scientific research documents, whether they are published or not. The documents may come from teaching and research institutions in France or abroad, or from public or private research centers.
L'archive ouverte pluridisciplinaire HAL, est destinée au dépôt et à la diffusion de documents scientifiques de niveau recherche, publiés ou non, émanant des établissements d'enseignement et de recherche français ou étrangers, des laboratoires publics ou privés. 


\section{Author's Accepted Manuscript}

Light-dependent orientation responses in animals can be explained by a model of compass cue integration

Kenneth Kragh Jensen

PII: S0022-5193(09)00425-1

DOI: doi:10.1016/j.jtbi.2009.09.005

Reference: YJTBI 5696

To appear in: $\quad$ Journal of Theoretical Biology

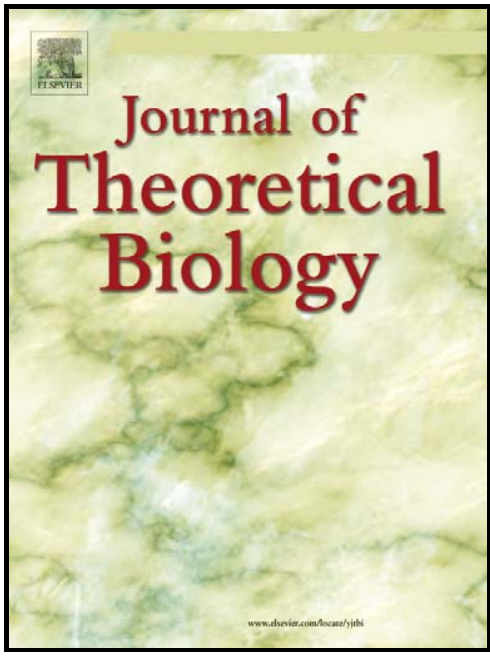

www.elsevier.com/locate/yjtb

Received date: 7 February 2008

Revised date: 2 September 2009

Accepted date: 8 September 2009

Cite this article as: Kenneth Kragh Jensen, Light-dependent orientation responses in animals can be explained by a model of compass cue integration, Journal of Theoretical Biology, doi:10.1016/j.jtbi.2009.09.005

This is a PDF file of an unedited manuscript that has been accepted for publication. As a service to our customers we are providing this early version of the manuscript. The manuscript will undergo copyediting, typesetting, and review of the resulting galley proof before it is published in its final citable form. Please note that during the production process errors may be discovered which could affect the content, and all legal disclaimers that apply to the journal pertain. 
1 Light-dependent orientation responses in animals can be

2 explained by a model of compass cue integration

3

4

5

6

7 Kenneth Kragh Jensen

8

9 Institute of Biology, University of Southern Denmark, DK-5230 Odense M, 10 Denmark.

11

12

13 E-mail: kkj@jensenkk.net

14 Phone: 1-812-855-8709

15 Fax: 1-812-855-4436

16

17

18 Present address: Indiana University, School of Medicine, Jordan Hall, 1001 East

19 Third Street, Bloomington, IN 47405, USA

21 


\section{Abstract}

The magnetic compass sense of animals is currently thought to be based on light-

23 dependent processes like the proposed radical pair mechanism. In accordance, many

24 animals show orientation responses that depend on light. However, the orientation

25 responses depend on the wavelength and irradiance of monochromatic light in rather

26 complex ways that cannot be explained directly by the radical pair mechanism. Here, a

27 radically different model is presented that can explain a vast majority of the complex

28 observed light-dependent responses. The model put forward an integration process

29 consisting of simple lateral inhibition between a normal functioning, light-independent

30 magnetic compass (e.g. magnetite based) and a vision based skylight color gradient

31 compass that misperceives compass cues in monochromatic light. Integration of the

32 misperceived color compass cue and the normal magnetic compass not only explains most

33 of the categorically different light-dependent orientation responses and their sequential

34 occurrence, but also shows a surprisingly good fit to how well the animals are oriented (r-

35 values) under light of different wavelength and irradiance. The model parsimoniously

36 suggests the existence of a single magnetic compass in birds (probably based on magnetite)

37 and explains the light-dependency as simple interference with another, vision based

38 compass.

41 Keywords: magnetoreception; monochromatic light; light-dependent orientation responses;

42 radical pair mechanism; magnetite. 


\section{Introduction}

Many animals use a number of different compass cues to orient during migration. It

46 has long been known that several animals can sense the Earth magnetic field and use it for

47 orientation (Wiltschko and Wiltschko, 1995a). However, after more than 30 years of

48 research it is still largely a puzzle exactly how the magnetic sense works and where the

49 sense organ is located. Two major competing hypotheses exist today on a sensory

50 transduction mechanism: one is based on small iron based magnetic crystals (IBMC) like

51 magnetite and the other a chemical compass based on magnetic properties of radical pairs

52 excited by light called the radical pair mechanism (see reviews in e.g. Johnsen and

53 Lohmann, 2005; Johnsen and Lohmann, 2008; Wiltschko and Wiltschko, 2005).

The transduction mechanism based on IBMC is generally hypothesized to consist of

55 the interaction of the Earth magnetic field with the magnetic crystals which then induce

56 stress and strain on associated membranes with mechanosensitive ion channels (see e.g.

57 Johnsen and Lohmann, 2005; Johnsen and Lohmann, 2008; Solov'yov and Greiner, 2008;

58 Walker, 2008). In vertebrates, there is good support of the existence of such an IBMC

59 compass system in the nasal region of rainbow trout (Oncorhynchus mykiss) (Diebel et al.,

60 2000; Walker et al., 1997) and in the skin of the upper beak and nasal region of a number of

61 birds (Beason and Nichols, 1984; Fleissner et al., 2007; Fleissner et al., 2003; Hanzlik et

62 al., 2000; Williams and Wild, 2001; Winklhofer et al., 2001). Several theoretical

63 considerations support that IBMC can function as a magneto reception system with no

64 major problems (Davila et al., 2003; Davila et al., 2005; Edmonds, 1992; Fleissner et al., 
2007; Kirschvink and Gould, 1981; Kirschvink and Walker, 1985; Shcherbakov and Winklhofer, 1999; Solov'yov and Greiner, 2007; Solov'yov and Greiner, 2008; Walker, 2008; Winklhofer et al., 2001; Yorke, 1979; Yorke, 1981; Yorke et al., 1985).

The radical pair mechanism is hypothesized to consist of light induced radical pairs that due to their spin configuration obtain magnetic moments. The recombination of the radical pairs may then be affected by the orientation of the molecules relative to the Earth magnetic field (Ritz et al., 2000; Schulten, 1982; Schulten et al., 1986). Currently, the major candidate molecules are the cryptochromes which are known to serve functions mainly in circadian rhythms (Ritz et al., 2000; Sancar, 1999; Sancar, 2003). Many conditions must be met if such a reaction is going to be sensitive to the weak Earth magnetic field, but it may not be impossible and theoretical considerations support the principles of the radical pair mechanism as a basis for magnetoreception (Adair, 1999; Cintolesi et al., 2003; Efimova and Hore, 2008; Maeda et al., 2008; Rodgers and Hore, 2009; Solov'yov and Schulten, 2009; Solov'yov et al., 2007; Wang et al., 2006; Weaver et al., 2000).

$$
\text { The intriguing fact that the orientation response of many animals is indeed }
$$
dependent on light has so far been taken as major support of the radical pair mechanism (for reviews see e.g. Johnsen and Lohmann, 2008; Wiltschko and Wiltschko, 2006; Wiltschko and Wiltschko, 2002). However, the observed light-dependency of orientation responses is rather complex and the experiments have left us with quite a puzzle to explain: depending on the wavelength and irradiance of monochromatic light the animals may orient in the normal expected direction, shift their orientation by $90^{\circ}$ relative to that direction, orient towards some unexplainable fixed direction, or be completely disoriented in spite of 
being perfectly able to see (Johnsen et al., 2007; Wiltschko and Wiltschko, 2006). Such complex responses do not follow directly from the radical pair mechanism. Furthermore, there are cases of both normal and shifted orientation responses at longer wavelengths (Muheim et al., 2002b; Wiltschko et al., 2004b) where cryptochromes have limited to no absorption (Johnsen et al., 2007) and there are cases of disorientation at wavelengths well within the cryptochrome's absorption spectrum (Wiltschko et al., 2007a; Wiltschko et al., 2003). Therefore, there might be an alternative explanation to the light-dependency. In addition to a magnetic compass many animals use celestial compass cues like the stars, the position of the sun, skylight polarization patterns and color gradients (Wiltschko and Wiltschko, 1999a). Obviously, a celestial compass involves vision and light. If such a sensory system is based on a system of photoreceptors that have different spectral sensitivities it may result in unexpected outputs in the highly unnatural stimulus of monochromatic light stimulates only subsets of the photoreceptors.

Multiple compass cues show clear evidence of being integrated into a single general system and specific compass mechanisms are thought not to be entirely independent from others (Wiltschko et al., 1994). Here, a model is proposed that provides a very different alternative explanation of the light-dependent orientation responses in animals. The model is cue integration based on simple lateral inhibition between "compass neurons" in compasses of different sensory modes: a color gradient compass and a magnetic compass. The integration is proposed to normally serve to increase the reliability and precision of an overall compass (Wiltschko et al., 1994). With propositions on how a color gradient sensory system may work, the model can explain a great deal of the observed complex pattern of light dependent orientation responses. In addition, the model predicts, 
111 surprisingly well, the concentration of the orientation responses (r-values) as a function of

112 light wavelength or irradiance. Contrary to the current view of a dual magneto sensory

113 system (IBMC and radical pair) the model parsimoniously suggests that animals have only

114 a single magnetic field sensory system (most likely is based on IBMC) and gives the

115 simplest explanation yet of the complex patterns of light-dependent orientation responses in 116 animals.

\section{Methods}

First, simplified models of a magnetic and a color gradient compass will be

119 proposed. Then an integration process between two such compasses will be put forward.

120 Propositions will then be made on how such a color gradient compass might lead to

121 misperceptions in monochromatic light of different wavelengths and irradiances. Finally, it

122 will be demonstrated how the integration model behaves in monochromatic light when such

123 an abnormal output of the color gradient compass is integrated with the normal output from

124 an intact magnetic compass.

\section{A magnetic compass}

126 An orientation cue is some geophysical or celestial parameter that can be used for

127 discriminating geographical directions. Let's begin by assuming a strongly simplified

128 hypothetical magnetic compass sensory system consisting of a circular array of compass

129 cells with the axis of each cell body pointing in a different geographical direction (Fig. 1A)

130 (as proposed by Deutschlander et al., 1999; Phillips and Borland, 1992a). A specific

131 transduction mechanism is not essential to the model and several suggestions can be found

132 elsewhere (see introduction for references). So let us just assume without any detail of a 6 
133 transduction mechanism that the compass cells with their cell axis aligned parallel to the

134 magnetic field lines along the magnetic North-South axis are maximally excited, resulting

135 in maximal neural spike frequencies. Oppositely, the cells having their axes aligned along

136 the East-West axis, perpendicular to the magnetic field, are minimally excited and fire with

137 some spontaneous spike frequency. Thus there will be a non-uniform circular distribution

138 of spike frequencies among the cells along all $360^{\circ}$ that has its maximum along the

139 magnetic North-South axis and minimum along the East-West axis. A simple non-uniform

140 circular distribution is the elliptic form. Let us therefore assume that the spike frequencies,

$141 S F$, as a function of geographical direction, $\omega$, follow a simple elliptic form and can be

142 represented mathematically by a standard ellipse equation as:

143

$$
S F(\omega)_{\operatorname{mag}} \approx \frac{A_{\operatorname{mag}} \cdot \theta_{\operatorname{mag}}}{\sqrt{A_{\operatorname{mag}}{ }^{2} \cdot \operatorname{cog} \varepsilon^{2}(\alpha)+\theta_{\operatorname{mag}}{ }^{2} \cdot \sin ^{2}(\omega)}}
$$

(Equation 1)

146 where the constants $A_{\text {mag }}$ and $B_{\text {mag }}$ represents the average spike frequency along the

147 maximal excited and the minimal excited axis respectively corresponding to the major and

148 minor axis of an ellipse. In the following the major and minor axes $A$ and $B$ will be referred

149 to as the major and minor compass axis, respectively. $S F(\omega)_{\text {mag }}$ can then be represented as

150 radial values in a polar plot as shown innermost in Fig. 1A with the radius in any given

151 direction corresponding to the average spike frequency of the cells with their axis pointed

152 in that direction. Larger radial values correspond to higher spike frequencies. The spike 
153 frequency in any given direction will be referred to as the radial spike frequency. The

154 circular distribution of spike frequencies will be referred to as the directional firing pattern.

155 A color gradient compass

156 The position of the sun is an important cue used by many animals for orientation

157 (see e.g. Wiltschko and Wiltschko, 1993). Scattering of the sunlight in the atmosphere

158 results in polarization patterns, intensity, and color gradients that are all dependent on the

159 position of the sun and can be used to reveal its position when it is not directly visible. The

160 color gradients consist of an increased amount of long wavelength light relative to short

161 wavelength/UV light in the direction of the sun and, oppositely, an increased amount of

162 short wavelength/UV light relative to longer wavelengths in the anti-solar direction,

163 whereas there is a more balanced distribution at an angle of $90^{\circ}$ relative to the direction of

164 the sun (Fig. 1B) (Coemans et al., 1994). Although it is known that key navigators like bees

165 and ants are able to use color gradients for rather precise orientation (Rossel and Wehner,

166 1984; Wehner, 1997), there has been surprisingly little focus on animal orientation by color

167 gradients . Given the simplicity and apparent efficiency of color cues, it is thus not unlikely

168 that color gradients are used by a wide number of animals for orientation including the

169 passerine birds. A model color gradient compass may be based on a simple contrast

170 between UV and long wavelength light:

171

172

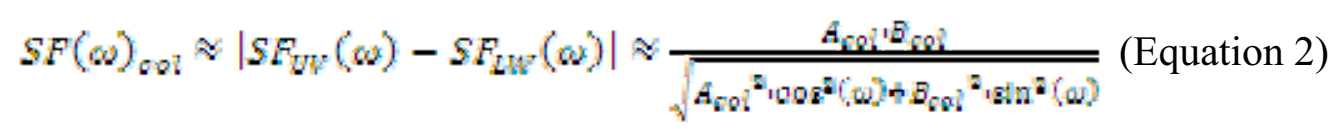


$174 S F\left(W_{100}\right.$, in any given direction, is the absolute difference between the spike frequency

175 elicited by a UV photoreceptor mechanism, $S F_{U V}(\omega)$, and that of a long wavelength

176 sensitive photoreceptor mechanism $S F_{L W}(\omega)$. $S F(\omega)_{c o l}$ would then be maximal $\left(A_{c o l}\right)$ along

177 the solar-anti solar axis (solar meridian) where the spectral contrast is maximal and

178 minimal along the axis $90^{\circ}$ relative to the solar meridian where the contrast is minimal

$179\left(B_{c o l}\right)$. Therefore, as for the magnetic compass, we will assume that there exists a circular

180 array of color gradient compass cells which will show a simple elliptic form of the

181 directional firing pattern (see Fig. 1B).

182 It has been suggested that light in the blue or mid wavelength range may serve as

183 some sort of reference color in a color gradient compass (Kinoshita et al., 2007). In that

184 case Eq. 2, which will be returned to later, becomes:

185

186

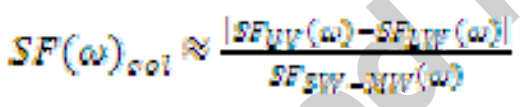

(Equation 3)

188 where $S F_{S W-M W}$ is the spike frequencies elicited by a photoreceptor mechanism in the short 189 and/or mid wavelength range. A high input from the SW - MW range relative to both the

190 UV and the LW range would then tend to inhibit the color compass spike frequency.

\section{Model compass integration}

192 Animals have a multitude of compasses based on the different celestial and 193 geophysical cues and it is thought that these multiple compasses are closely integrated into 194 an overall compass (Able, 1991; Wiltschko et al., 1994). Lateral inhibition, where 
195 neighboring neurons have a mutual inhibitory effect, is common in many sensory systems

196 like for instance in vision contrast enhancement (Golden, 1996). Lateral inhibition is likely

197 to occur in compass sensory systems too and could for instance function as a contrasting

198 effect between cues ensuring that the most prominent compass cue in any given direction

199 dominates. The current model will therefore assume that compass cues are integrated

200 through lateral inhibition and the resultant integrated compass is assumed to be simply the

201 absolute value of the difference in spike frequency of the two above compasses as a

202 function of direction as shown in the following equation:

203

207 of 100 spikes/s along the geographical North-South axis, $A_{c o l}=100$, and let us say that the

208 minor compass axis, the spontaneous spike frequency, is approximately one third of this, 35

209 spikes/s, along the East-West axis $\left(B_{c o l}=35\right)$. Let us assume the hypothetical magnetic

210 compass has the same spontaneous spike frequency of 35 spikes/s along the East-West axis

$211\left(B_{m a g}=35\right)$ but because celestial cues appear to be dominant and preferred over magnetic

212 cues (Mouritsen, 1998; Muheim, 2006; Wiltschko et al., 1994) the precision of the

213 magnetic compass is assumed lower and the maximal spike frequency along the North-

214 South axis will be assigned to be, for instance, $70 \%$ of the celestial compass $\left(A_{\text {mag }}=70\right)$.

215 Fig. 2A then shows the result of the integration (solid line; Eq. 4) of the color gradient

216 compass (dashed line) and the magnetic compass (dotted line). As can be seen, the result of 
217 the integration is a compass with a higher contrast between the North-South and East-West

218 axes. Equally important, the compass that is maximally excited will dominate in a situation

219 where there, for instance, is a moderate mismatch between the two compasses (Fig. 2B).

220 For simplicity, the model does not consider time compensation for celestial rotation

221 in the color gradient compass or declination between the color gradient compass and

222 magnetic compass (i.e. declination $=0$ ). The major compass axes of the two compasses will

223 thus always be aligned and both compass cues point out the same North-South axis. The

224 animal's normal orientation is assumed to be towards that common compass North. North

225 may be separated from South based on inclination in the magnetic compass (Wiltschko,

226 1972), and for the color gradient compass, the solar direction may be separated from the

227 anti-solar direction by its maximal content of long wavelength light under natural

228 conditions. Finally, we will assume that the animals do not assess the cues from each

229 compass separately in a normal orientation context and that they will uncritically orient

230 along the axis of maximal excitation of the integrated compass only (this may be different

231 in a calibration context).

\section{Behavior of color gradient compass in monochromatic light}

233 It follows from Eq. 2 that if the incoming light is monochromatic and excites

234 predominately the UV or the LW mechanism, the radial spike frequency of the color

235 gradient compass, $S F(\omega)_{\text {col }}$, will be high but since the light comes from all directions, the

236 directional firing pattern will be uniform and form a circle rather than an ellipse (i.e. $A_{c o l}=$

$237 B_{c o l}$; Fig. 1C). The higher the irradiance of monochromatic light exciting predominately one

238 color detector, the larger the $\left|S F_{V W}-S F_{U W}\right|$ difference in all directions and thus the higher 
239 the radial spike frequency $S F(\omega)_{c o l}$ (i.e. larger radius of the circular directional firing

240 pattern). Similarly, the more the wavelength of the monochromatic light skews towards and

241 predominately excites one color detector over the other, the larger the $\left|S F_{W V}-S F_{U W}\right|$

242 difference and thus the higher the radial spike frequency $S F(\omega)_{c o l}$. In the following

243 treatment, the term $S F(\omega)_{c o l}$ will be referred to as $S F(\omega)_{o o l}^{\prime}$ as a reminder that the

244 directional firing pattern is uniform and forms a circle and that its radial spike frequency

245 ("radius") depends on the irradiance and wavelength of the monochromatic light.

246 Behavior of integration in monochromatic light

247 What happens if we do the integration with the uniform directional firing pattern

248 suggested above? Fig. 3A shows an initial case of compass integration where the

249 monochromatic light is assumed to excite predominately one color detector but where the

250 irradiance is low and results in a low radial spike frequency of the color gradient compass.

251 Here there is little effect of the color gradient compass on the integrated compass and the

252 animals will be expected to orient normally towards the North. As the irradiance of the

253 monochromatic light increases the radial spike frequencies of the color gradient compass,

$254 S F(\omega)_{\circ o l}^{\prime}$, will increase. At one point the integrated output will have four lobes and be

255 equally excited in both the North-South and East-West direction and the animals will be

256 expected to be disoriented (Fig. 3B). As the light irradiance increases further the East-West

257 axis will start to dominate and the animals will be expected to shift their orientation by $90^{\circ}$

258 (Fig. 3C). Then, as the light irradiance is increased further the animals will keep showing

259 shifted orientation, but as can be seen, the precision of the compass will gradually degrade 12 
260 and the orientation will be poorer and poorer until the animals finally show disorientation

261 again (Fig. 3D-E). An animated sequence of the integrated compass output as a function of

262 the model color gradient compass input is shown in Supplemental Movie 1.

263 A similar pattern of orientation responses is to be expected if we instead keep the

264 irradiance of the monochromatic light constant and vary the wavelength. There will be no

265 output from the color gradient compass if, initially, the wavelength of the monochromatic

266 light lies within a range where the input to the UV and long wavelength mechanism is

267 balanced. However, if we then shift the wavelength of the monochromatic light towards the

268 sensitivity of the UV or the LW receptors, then the radial frequency, $S F(\omega)_{\circ o l}^{\prime}$, will

269 increase. Therefore, the more the wavelength of the monochromatic light moves towards

270 the spectral sensitivity of the UV or the long wavelength receptors, the more the radial

271 spike frequencies, $S F(\omega)_{\odot \circ i}^{\prime}$, increase and (if the irradiance is high enough) we will expect

272 to observe the exact same sequence of orientation responses going from normal (Fig. 3A),

273 to disoriented (Fig. 3B), to a $90^{\circ}$ shift (Fig. 3C), and again towards disoriented (Fig. 3D-E).

274 Model predictions of a mosaic pattern of orientation responses

275 It has now been shown how the hypothetical integration process is influenced as a

276 function of the relative excitation level of the color gradient compass photoreceptors. Let us

277 now consider what categories of orientation responses the model predicts to be distributed

278 around the spectral sensitivities of the color detectors as a function of both wavelength and

279 intensity of the light. Let us assume that we have a color gradient compass of the type

280 proposed above with a UV and a LW detector (Fig. 4). Let us first assume that animals in 
281 an orientation test are placed under illumination of monochromatic light of constant

282 wavelength close to the peak spectral sensitivity of either the UV or long wavelength

283 detectors (arrows 1). Under very low irradiances the output from the color gradient

284 compass will be minimal and not affect the orientation (black " $N$ "). As the irradiance is

285 increased the animals will show the predicted sequence of orientation responses (c.f. Fig. 3

286 and see above) going from normal orientation, to disorientation (light gray "D"), to $90^{\circ}$

287 shift (darker grey "S"), and towards disorientation again. Oppositely, if the wavelength of

288 the monochromatic light lies between the peak spectral sensitivities of the UV and LW

289 detectors in a range where they are equally excited, then $S F(\omega)_{\sigma o:}^{\prime}$ will be constantly low

290 and the orientation will stay normal and independent of irradiance (arrow 2).

291 Let us instead keep the irradiance constant and vary the wavelength (double arrow

292 3). If the wavelength is at first kept between the UV and LW receptors (middle of arrow 3)

293 the orientation will be normal as mentioned above, but as we change the wavelength

294 towards either the UV or LW detectors then these will start being increasingly excited

295 relative to the other detector and the model predicts an identical sequence of orientation

296 responses with responses going from normal, to disoriented, to shifted, and back to

297 disoriented.

298 The further the wavelength of the monochromatic light is placed towards the

299 spectral sensitivity of the UV or LW detectors, the lower the irradiance of the

300 monochromatic light is necessary to excite that detector a certain amount relative to the

301 other detector. We will therefore expect that the further the wavelength of the

302 monochromatic light is skewed towards the spectral sensitivity of one of the detectors, the 
303 lower the irradiance is necessary to elicit a given orientation response and the response

304 sequence $(N \rightarrow D \rightarrow S \rightarrow D)$ will start at lower and lower irradiances. This is illustrated by

305 arrows 4 where, if we for instance fix the attention to the shifted orientation response (S),

306 we will expect this particular response to occur at lower and lower irradiances as we go

307 towards the spectral sensitivity of the UV or LW detectors. The proposed relationship

308 between orientating responses and the absorption spectra of the detectors is highly

309 suggestive and the exact relationship must depend on factors like the specific form of the

310 detector's absorption spectra, the relative sensitivity of the detectors, and the overlap

311 between the detectors.

312 Predictions on the concentration of orientation (r-value)

313 The r-value is used in circular statistics as a measure of the concentration of

314 individual headings around the mean direction (Batschelet, 1981). An r-value of 1 indicates

315 that the experimental animals are all headed in the exact same direction. An r-value of 0

316 indicates that the animals were totally disoriented and headed with equal probability

317 towards any direction. The precision by which a group of animals head towards a common

318 goal must strongly depend on the precision of their available compasses. It is fair to assume

319 for our hypothetical array of compass neurons that the more excited the cells coding the

320 North-South axis are relative to the cells coding the East-West axis, the more precise the

321 overall compass works and the better the animals are expected to be oriented. A common

322 measure of the relative sizes of the axes in an ellipse is the eccentricity. The eccentricity for

323 a compass will be termed the compass eccentricity, $\varepsilon_{c}$, and is defined as follows: 


$$
s_{0}=\sqrt{1-\frac{D_{s}^{2}}{A_{0}^{2}}} \quad \text { (Equation 5) }
$$

$327 A_{c}$ and $B_{c}$ are the major and minor compass axes of the given compass (see above). The

328 eccentricity varies between 0 and 1 with 0 indicating a circle and 1 an extreme oval form. A

329 compass is useless in pointing out directions and cause disorientation and r-values near 0

330 when the major and minor compass axes are equal $\left(A_{\varepsilon}=B_{\varepsilon}\right)$ and $\varepsilon_{c}=0$ and will have an

331 optimal precision and cause strong directional headings with r-values near 1 when $A_{\sigma} B_{\sigma}$

332 and $\varepsilon_{c}$ goes towards 1 . Thus we expect r-values and compass eccentricities to be highly

333 correlated.

334 Fig. 5 shows how the compass eccentricity of the integrated compass changes as a

335 function of $S F(\omega)_{\circ o l}^{\prime}$ (calculated as in Fig. 3 with $A_{\text {mag }}=70$ and $B_{\text {mag }}=35$ for the magnetic

336 compass). Fig. 5 also contains references to each of the examples in Fig. 3 (A-E) where the

337 directional firing pattern of the integrated compass can be seen for comparison. It should be

338 noted that the directional firing pattern of the integrated compass is obviously more

339 complex than the elliptic form and the compass eccentricity, which is defined for an ellipse,

340 strictly does not apply. However, this treatment seems quite adequate for the current

341 purpose and still gives a good measure of the contrast between the North-South and East-

342 West axis of the compass. 


\section{Fitting compass eccentricity to observed r-values}

344 As mentioned above the compass eccentricity, $\varepsilon_{c}$, is expected to be highly correlated

345 with r-values and it is predicted that r-values in the literature will follow a similar pattern to

346 that of the compass eccentricity in Fig. 5. Therefore, in the following, fits of Eq. 5 as a

347 function of $S F(\omega)_{\odot \circ l}^{\prime}$ will be made to r-values in the literature. However, in practice there

348 is a problem in the fact that the compass eccentricity in Eq. 5 cannot easily be used as a

349 fitting function in a simple mathematical way because when the integration compass shifts

$35090^{\circ}$ as $B_{\text {int }}>A_{\text {int }}$ we end up with the square root of a negative number. To solve this, the

351 following equation has been developed which does not have that problem and comes very

352 close to the conventional eccentricity measure:

353

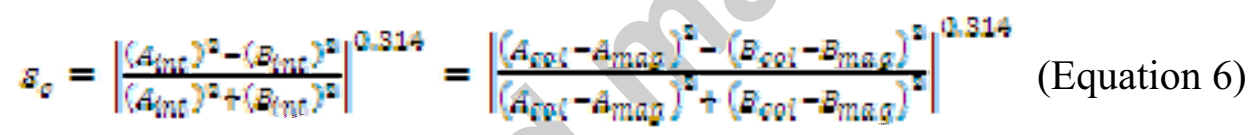

356 The parameter 0.314 was found by iterative fitting of Eq. 6 to curves of Eq. 5 (see Fig. A1

357 in the appendix).

358 Scaling along both the abscissa and ordinate is necessary in order to fit the

359 theoretical compass eccentricities to the wavelength and irradiance data ranges of the real r-

360 values. This will be done by including some simple scaling parameters to Eq. 6. It turns out

361 that scaling along the abscissa provides a better fit to the irradiance and wavelength scale

362 when this is done by exponential transformation whereas it suffices with simple 
363 multiplication along the ordinate. Therefore, the literature data will be fitted by the

364 following equation:

365

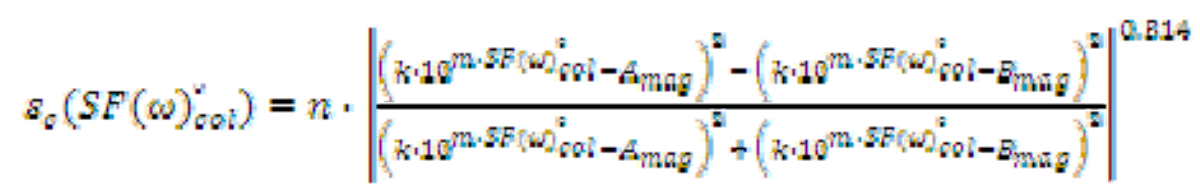

368 The parameter $n$ is then the linear multiplication factor for scaling along the ordinate and $k$

369 and $m$ are the parameters for exponential scaling along the abscissa. The parameters of the 370 model magnet compass were kept constant during the fitting procedure with $A_{m a g}=70$ and

$371 B_{\text {mag }}=45$. These constant values of the magnetic compass seemed to give the best fits and 372 were determined initially through fitting procedures with $B_{m a g}$ as an additional parameter.

373 The effect of changing the various fitting parameters is shown in Fig. A2 of the appendix. 374 As an indication of the goodness of fit the adjusted correlation coefficient $R_{a d j}$ is 375 reported in the results. $R_{a d j}$ compensates for the number of parameters and the replication 376 and is lower than the standard correlation coefficient (Zar, 1996). P-values (F-test) of the 377 significance of the overall fit are also reported. It is important the reader keep in mind that 378 although Eq. 7 may look involved it represents in reality a simple measure of the 379 eccentricity (Eq. 5/6) stretched or shrunk along both axes to adapt to and fit the range of 380 real r-values. The fitting procedure was performed in Origin 8 Pro with Eq. 7 added as a 381 custom non-linear function.

\section{Results}




\section{Categorical orientation responses}

Fig. 6 shows the three categorical orientation responses (normal, shifted, and

385 disorientation) reported in the literature as a function of wavelength and irradiance. Cases

386 of normal orientation are shown with black triangles, cases of shifted orientation are shown

387 as open diamonds with a cross line, and cases of disorientation are shown as open circles.

388 Overlapping data is shown by a cross at the original data point and the number of actual

389 data is shown as dots on both sides of the cross. Fixed uni-modal responses has been taken

390 as shifted orientation if they deviated from the normal direction by more than $45^{\circ}$ and as

391 normal if they deviated by less.

392 The arrows $1-4$ in Fig. 6 suggest the very same trends exist in the real orientation

393 data as those predicted by the model (c.f. Fig. 4; for ease of reference with Fig. 4 the arrows

394 in Fig. 6 have been given the same categorical numbers (1-4)). Let us first look at cases

395 where the wavelength is constant and the irradiance is changed. Along the right arrow

396 assigned " 1 " around $565 \mathrm{~nm}$ the birds were seen to be predominately normally oriented

397 below $20 \cdot 10^{15}$ quanta $/ \mathrm{m}^{2} / \mathrm{s}$, between 20 and $35 \cdot 10^{15}$ quanta $/ \mathrm{m}^{2} / \mathrm{s}$ they were predominantly

398 disoriented, from 35 to $55 \cdot 10^{15}$ quanta $/ \mathrm{m}^{2} / \mathrm{s}$ the birds were mainly shifted in their

399 orientation, and at approximately $70 \cdot 10^{15}$ quanta $/ \mathrm{m}^{2} / \mathrm{s}$ there was both a case of expected

400 disorientation and a single case of normal orientation not explainable by the model.

401 Similarly, along the left arrow marked "1" at $424 \mathrm{~nm}$ the birds were normally oriented

402 around $10 \cdot 10^{15}$ quanta $/ \mathrm{m}^{2} / \mathrm{s}$, predominantly disoriented between approximately 30 and 40 .

$40310^{15}$ quanta $/ \mathrm{m}^{2} / \mathrm{s}$, then shifted in orientation just above $40 \cdot 10^{15}$ quanta $/ \mathrm{m}^{2} / \mathrm{s}$, and

404 disoriented again around $55 \cdot 10^{15}$ quanta $/ \mathrm{m}^{2} / \mathrm{s}$ but a case of normal orientation around 70 .

$40510^{15}$ quanta $/ \mathrm{m}^{2} / \mathrm{s}$ not explainable by the model. In contrast, in tests around $500 \mathrm{~nm}$ the birds 19 
406 showed normal orientation at all intensities from just below $10 \cdot 10^{15}$ quanta $/ \mathrm{m}^{2} / \mathrm{s}$ up to 70 . $40710^{15}$ quanta $/ \mathrm{m}^{2} / \mathrm{s}$ with just a single deviant case with shifted orientation (arrow 2; c.f. Fig. 408 4).

409 Now let us keep the irradiance constant and see what the data show when the 410 wavelength is changed. Along the lowermost arrow assigned "3" in Fig. 6 at irradiances

411 around $10 \cdot 10^{15}$ quanta $/ \mathrm{m}^{2} / \mathrm{s}$ it can be seen that from around 500 to $565 \mathrm{~nm}$ the orientation 412 responses were predominantly normal, then around $590 \mathrm{~nm}$ the birds were all disoriented, 413 at approximately $620 \mathrm{~nm}$ they were shifted $90^{\circ}$ in their orientation, and between 625 and $414650 \mathrm{~nm}$ they were again predominately disoriented. Along the upper arrow assigned " 3 " at 415 an irradiance round $45 \cdot 10^{15}$ quanta $/ \mathrm{m}^{2} / \mathrm{s}$ the birds predominately showed normal 416 orientation around $500 \mathrm{~nm}$, there was no disorientation (possibly because of lacking data), 417 but around $565 \mathrm{~nm}$ the birds were predominately shifted in their orientation, and around $418600 \mathrm{~nm}$ and upwards the birds were disoriented. The two arrows marked "4" both suggest 419 that the sequence of orientation responses were skewed towards lower irradiances when 420 going from around $425 \mathrm{~nm}$ to $375 \mathrm{~nm}$ or from around $565 \mathrm{~nm}$ to around $625 \mathrm{~nm}$ as 421 predicted by the model (see above and Fig. 4). In conclusion, the passerine literature shows 422 a rather strong overall correlation in its pattern of orientation responses as a function of 423 both wavelength and irradiance to those predicted by the model.

424 A predicted sequence of shifts in categorical orientation responses at a constant 425 irradiance of $45 \cdot 10^{15}$ quanta $/ \mathrm{m}^{2} / \mathrm{s}$ (except $22 \cdot 10^{15}$ quanta $/ \mathrm{m}^{2} / \mathrm{s}$ at $400 \mathrm{~nm}$ ) was also seen 426 in data from the red-spotted newt (Tab. 1). The newts changed from normal orientation at 427400 and $450 \mathrm{~nm}$ to disorientation at $475 \mathrm{~nm}$ and finally a $90^{\circ}$ shift at $500 \mathrm{~nm}$. The data is 
428 too sparse in the other species shown in Tab. 1 to take into consideration, but does not 429 disagree with the model.

\section{Concentration of orientation}

Fig. 7 shows how r-values from the literature changed with irradiance at constant

432 wavelengths (Fig. 7A-B) or with wavelength at approximate constant irradiances (Fig. 7C-

433 D). Averages with standard deviation error bars are shown in cases where more than one

434 data point was available, but all fits were performed on individual data points (sample size

$435 \mathrm{~N}$ is given in the figures). At a constant wavelength of $424 \mathrm{~nm}$ the cue integration model

436 showed a good fit to available r-values with an $R_{a d j}$ value of 0.81 and $p=0.0004$ (Fig. 7A).

437 The point in the normal orientation category at highest irradiance (in brackets) seems to be 438 a significant outlier both in value and orientation category and it was excluded from the 439 analysis. Including the outlier gives the statistics $R_{a d j}=0.47$ and $p=0.04$ which still is 440 significant, but not surprisingly with a much lower $R_{\text {adj }}$ value. There is evidence that 441 polarization cues and possibly the correlated color gradients can be suppressed at high 442 irradiances (Kinoshita et al., 2007) and in such case, the model would actually predict 443 normal orientation. At a constant wavelength of $565 \mathrm{~nm}$ there was good fit of the model to 444 the r-values with $R_{a d j}=0.74$ and $p<0.0001$ (Fig. 7B). Again, the outlier at high irradiance 445 was excluded with the same argument as above. Inclusion of the outlier results in the 446 alternative statistics $R_{a d j}=0.67$ and $p<0.0001$ which still is a rather good overall fit. At 447 approximate constant irradiances a good fit was shown at an irradiance between $6-14$. $44810^{15}$ quanta $/ \mathrm{m}^{2} / \mathrm{s}$ with $R_{a d j}=0.93$ and $p<0.0001$ (Fig. 7C) and at an approximate constant 449 flux of $43-54 \cdot 10^{15}$ quanta $/ \mathrm{m}^{2} / \mathrm{s}$ with $R_{a d j}=0.74$ and $p<0.001$ (Fig. 7D). 
Data was also available for the red-spotted newt at a constant irradiance of $45 \cdot 10^{15}$

451 quanta $/ \mathrm{m}^{2} / \mathrm{s}$ (except $22 \cdot 10^{15}$ quanta $/ \mathrm{m}^{2} / \mathrm{s}$ at $400 \mathrm{~nm}$ ). If the outlier at $600 \mathrm{~nm}$ was included

452 in the analysis the fit is poor with $R_{\alpha d j}^{2}=-0.34$ and $p=0.016\left(R_{\sigma a j}^{2}\right.$ is negative if the fit is

453 very poor and the residual mean squares are larger than the total mean square (Zar, 1996)).

454 If the outlier was masked from the analysis a fair fit is obtained with $R_{a d j}=0.77$ and $p=$

455 0.004. Since the latter analysis is more informative the summary of this analysis was

456 included in Fig. 7E.

457 Finally, since the fitting procedure was done by simple scaling of a fixed curve

458 form, each data point can be transformed back into the numerical domain of the model

459 (spikes/s) independent of whether the data point originally is a function of irradiance or

460 wavelength. This way, the overall fit of the model to all available literature data points can

461 be assessed. As can be seen in Fig. 7F, the summarized overall fit to the total data was quite

462 remarkable, in spite of the fact that the data were from different bird species, different

463 studies, and/or originated as a function of irradiance or wavelength. Running a fit on the

464 total data set gives a $R_{a d j}=0.94$ and $p<<0.0001$.

465 Spectral correlations

466 If the underlying visual compass is a color gradient compass we would expect

467 disorientation and $90^{\circ}$ shifts in both the UV (or near UV) and longer wavelengths. In birds

468 (were most data is present) this is the case and in general fits a model based on color

469 gradient very well. The photoreceptors involved in passerines may be the UV sensitive

470 (UVS) cones and/or possibly the short wavelength sensitive (SWS) for the UV/near UV

471 mechanism (spectral sensitivities of typical passerine photoreceptors are shown in Fig. 6). 
472 The LW mechanism may be mediated by the mid wavelength sensitive (MWS), double

473 cones (DC), and/or long wavelength sensitive cones (LWS). If there is a reference color,

474 the SWS, MWS, and/or rods seem to be possible candidates.

475 In the newts the pattern does not immediately fit the idea of a color gradient

476 compass since orientation shifts are mainly observed in the MW-LW with no observations

477 near the UV (Table 1). However, the newt has not been as extensively studied with several

478 different irradiances as with the passerine birds, where it is evident, that very different

479 results are to be expected at different irradiances. Tests with higher irradiance and further

480 down in the UV may thus reveal patterns that allow a better comparison. Orientation by

481 color gradients has already been documented in insects and they would provide good test

482 animals for the model. Unfortunately, the light dependent orientation response data

483 available for insects (Tab. 1) are too few and can be interpreted in too many ways to allow

484 any meaningful statements.

485 Discussion

487 responses surprisingly well and provides an important alternative to the radical pair

488 hypothesis as an explanation of these responses. It combines the questionable dual

489 magnetic reception hypotheses (radical pair and magnetite) into one simple hypothesis that

490 parsimoniously points to a single magneto-reception mechanism (probably based on IBMC)

491 which through an integration process with a celestial compass can be interfered with by

492 light. The literature data support the proposed cue integration model very well: the

493 predicted sequence of categorical orientation shifts as a function of both irradiance and 
494 wavelength match closely to those observed in the literature (Fig. 6) and model predictions

495 on the concentration of orientation as a function of both irradiance and wavelength fits

496 surprisingly well with observed r-values (Fig. 7).

\section{Model performance}

498 Although it has great explanatory power the model is crude in its current form. It

499 has for instance a problem if both cues happen to be equal in all directions since the

500 integration here will cancel to zero. Future elaborations are necessary if the model can be

501 validated, but inappropriate to address further at the current stage. The model is very robust

502 and will give very similar predictions and good fits within a wide range of the parameters

$503 A_{m a g}, B_{m a g}$, and $S F(\omega)_{\odot \circ !}^{\prime}$ that are far from limited to the arbitrary ones used. The model is

504 not dependent on the elliptic form of $S F_{m a g}(\omega)$ and will give very similar results with

505 integration between any elongated form and the uniform circular distribution of $S F(\omega)_{\circ \circ t}^{\prime}$.

506 The model is based on an assumption of lateral inhibition. Pragmatically, lateral inhibition

507 is more complicated than simple subtraction (Golden, 1996). However, the model is robust

508 and as long as the lateral inhibition function is linear and uniform in all geographical

509 directions the same overall model pattern will emerge and result in similar predictions.

\section{$510 \quad$ Future test of model validity}

511 In the following I will present some suggestions on how to test the cue integration

512 model with focus on the passerine birds. Unfortunately, since the presence and nature of a 
513 color gradient sensory system is unknown in vertebrates it is difficult to suggest any direct

514 test of the model.

\section{The presence of a color gradient compass}

516 First of all it will be important to establish if birds sense and are able to orient by

517 skylight color gradients. This should be relatively easy to establish for instance by placing

518 birds in the traditional test funnels covered by a frosted dome and set up color gradients by

519 differential illumination. However, great care must be taken to exclude intensity cues

520 (Coemans and Vos, 1992). The magnetic field has to be cancelled by Helmholtz coils to

521 eliminate it as a compass cue.

522

523

524

525

526

527

528

529

530

531

532 533

\section{Illumination with two or more monochromatic sources}

The idea of testing birds in illumination by two or more different monochromatic light sources is a good one (Wiltschko et al., 2004a). Unfortunately, the results of Wiltschko et al (2004a) are difficult to interpret and the birds behaved different in autumn and spring. However, such types of experiments could be done to address some key characteristics of the cue integration model. There seems to be at least three different possible constitutions of a color gradient compass: 1) a compass that works without a reference color and is a simple UV/LW antagonism (Eq. 2; compass 1 in Tab. 2). 2) A compass that has UV/LW antagonism but uses a color in the SW-LW range as a reference (Eq. 3; compass 2 in Tab. 2) . 3) A color gradient compass with no UV/LW antagonism and with a color in the SW-MW range as a reference (compass 3 in Tab. 2): 


$$
S F(\omega)_{o o t} \approx \frac{\left|S E_{W V}(\omega)+S E_{\omega W}(\omega)\right|}{S F_{S W}-M W(\omega)} \quad \text { (Equation 8) }
$$

537 experimental outcomes in the mixed color conditions as shown in Tab. 2: Experiment 1a:

538 the initial condition in Exp. 1a is at a constant irradiance in the UV (around perhaps 375

$539 \mathrm{~nm}$ ) high enough to elicit disorientation (the final stage of disorientation (end of arrows 1 in

540 Fig. 4)). This is probably occurring at a constant illumination of $20-30 \cdot 10^{15}$ quanta $/ \mathrm{m}^{2} / \mathrm{s}$.

541 In case of compass 1 and 2 the UV/LW antagonism will increasingly counteract each other

542 as the LW irradiance $(\sim 625 \mathrm{~nm})$ is increased and the orientation will be predicted to change

543 from disorientation into shifted orientation, to disorientation, and eventually when the UV

544 and LW color antagonism cancel each other, the orientation becomes normal. The normal

545 orientation is therefore expected to happen when the sensitivity of the LW mechanism

$546\left(\mathrm{~S}_{\mathrm{LW}}\right)$ approximates that of the UV mechanism at $20-30 \cdot 10^{15}$ quanta $/ \mathrm{m}^{2} / \mathrm{s}\left(\mathrm{S}_{\mathrm{UV} .20-30}\right)$ and

547 will give an estimate of the relative sensitivities of the UV and LW mechanisms. In the case

548 of compass 3 with no UV/LW antagonism the orientation response is expected to be

549 constant disorientation. Experiment 1b: in case of compass 1 and 2 the opposite sequence

550 of orientation responses $(N \rightarrow D \rightarrow S \rightarrow D)$ of Exp. 1a is expected to be observed as the

551 LW irradiance is increased from $S_{L W}=S_{U V .20-30}$ to $S_{L W}=2 \cdot S_{U V .20-30}$ (given the system is

552 linear). However, disorientation is again expected throughout for compass 3 . Experiment

553 2: in case of compass 1 and 2 , the initial condition here is the one found in Exp. 1 where the

554 sensitivity of the LW mechanism is the same as that of the UV mechanism at $20-30 \cdot 10^{15}$

555 quanta $/ \mathrm{m}^{2} / \mathrm{s}\left(S_{L W}=S_{U V .20-30}\right)$ and the orientation is normal. If the irradiance in the SW-MW 
range $(\sim 500 \mathrm{~nm})$ is increased the orientation will stay normal for compass 1 and 2 since the

557 UV and LW mechanisms will always be cancelled out. However, for compass 3 with no

$558 \mathrm{UV} / \mathrm{LW}$ antagonism, the orientation will be expected to be disorientation in the initial

559 condition, but expected to show a $D \rightarrow S \rightarrow D \rightarrow N$ sequence of orientation responses as

560 the increasing SW-MW irradiance increasingly reduces $S F(\omega)$ ool (obviously it is not

561 possible to establish $S_{L W}=S_{U V .20-30}$ as with compass 1 and 2 for compass 3, and here, an

562 illumination of $15 \cdot 10^{15}$ quanta $/ \mathrm{m}^{2} / \mathrm{s}$ in both the UV and LW will probably be suitable and

563 result in disorientation). Experiment 3: here all compasses are predicted to show an initial

564 orientation response of disorientation. As the SW-MW irradiance is increased compass 1

565 predicts constant disorientation since it has no reference color and the UV/LW contrast will

566 stay constant. Oppositely, compasses 2-3 with reference detectors will be expected to show

567 the $D \rightarrow S \rightarrow D \rightarrow N$ sequence. Experiment 4: seems trivial and will not help separate the

568 compasses, but predicts that the typical normal orientation observed at irradiances of, for

569 instance, 15 quanta $/ \mathrm{m}^{2} / \mathrm{s}$ (Fig. 6) is not due to a mechanism that depends on this wavelength

570 to function for normal magneto reception and that once saturated will continue to function

571 normally regardless of the presence of other wavelength light (as would be the logical

572 consequence of a cryptochrome based radical pair magnetic compass), but is part of a

573 system that is dependent on the relative balance between wavelengths as is the case in the

574 proposed cue integration model.

575 As follows from the above and Tab. 2, performance of Exp. 1-4 can help establish

576 whether or not a group of test birds will show behaviors that are consistent with the model

577 predictions. Although it may not provide direct evidence, positive results will strongly 
578 support the cue integration model. Especially so if the existence of a color gradient

579 compass can be demonstrated at the same time. Exp. 1-3 will help determine what type of

580 compass (1-3) lies behind the integration process.

\section{A possible role of a polarization compass?}

Although the simplicity of the color gradient compass highly favors it as the major

583 candidate in the model, there is a theoretical possibility of an alternative role of a

584 polarization compass which deserves mentioning. In polarization sensitivity two

585 orthogonally oriented polarization receptors and a luminosity detector is sufficient to

586 determine the three important factors: total intensity $(I)$, e-vector orientation $(\varphi)$ and degree

587 of polarization $(d)$ (Bernard and Wehner, 1977; Flamarique and Hawryshyn, 1998; Wehner,

588 1983; Wehner et al., 1975). If the detectors have separate spectral sensitivities, a compass

589 based on the degree of polarization and/or e-vector orientation may lead to misperceptions

590 similar to those proposed for the color gradient compass. In support of this, polarization

591 vision in many animals may not always be entirely independent of color. In vertebrates,

592 some fishes show clear evidence of the use of different color sensitive cones (UV and LW

593 sensitive) in polarization vision (Cameron and Easter, 1993; Coughlin and Hawryshyn,

594 1995; Hawryshyn and McFarland, 1987; Parkyn and Hawryshyn, 1993), although here, it

595 has been concluded that color is unlikely to confound with polarization sensitivity

596 (Ramsden et al., 2008). In invertebrates, Daphnia pulex show wavelength dependent

597 polarization vision and shift their orientation $90^{\circ}$ relative to the normal angular direction in

598 polarized light of wavelengths above $570 \mathrm{~nm}$, they are disoriented in polarized light of

599 wavelengths above $515 \mathrm{~nm}$, whereas they keep their orientation normal in polarized light of 
wavelengths above $455 \mathrm{~nm}$ (Flamarique and Browman, 2000). In the locust (Schistocerca

601 gregaria) polarization sensitive neurons respond differently to both unpolarized UV and

602 green light, and polarized blue light indicating different roles of spectrally different units in

603 polarization related vision (Kinoshita et al., 2007). Papilio butterflies show polarization

604 vision that is dependent on colors and anatomical and physiological data has been pointed

605 out to suggest an interdependence of polarization and color vision in other species like

606 Pieris and Nymphalid butterflies, tabanid flies (Tabanidae), ladybirds (Coccinella

607 septempunctata), and Waterstriders (Gerris palludum) (Kelber et al., 2001).

\section{Acknowledgements}

I dedicate this work to my parents Poul Jensen and Joan Kragh Jensen, to my two

610 kids Tinus Michel Kragh Jensen and Lara Michel Kragh Jensen, and to my wife Meredith

611 Leigh Jensen. I thank Ole Næsbye Larsen, Henrik Mouritsen, Rachel Muheim, and

612 Wolfgang Wiltschko for comments on earlier crude drafts in 2001 - 2002. I thank John B.

613 Phillips and Michael Walker for comments on more recent drafts in 2008. I thank Almut

614 Kelber and Uwe Homberg very much for valuable critique of parts related to vision. I thank

615 Meredith Leigh Jensen for language corrections. Finally I thank the three reviewers for

616 comments leading to great improvements of the manuscript. Part of this work was

617 performed while being supported by a grant from Carlsbergfondet (2006_01_0513) and a

618 grant from the Danish National Research Foundation awarded to the Center of Sound

619 Communication. 
622 figures (figures texts are below) that I think is an important elaboration on technical parts

623 of the fitting procedure to convince the reader that the fitting is in principle very simple and

624 that the parameters does not change the curve form per se.//

626 Figure A1: Comparison of the traditional eccentricity (Eq. 5) and the modified eccentricity

627 (Eq. 6). As can be seen the two curves come very close. The modified eccentricity was

628 introduced to automate and improve the fitting procedure by avoiding problems with square

629 roots of negative numbers when the model orientation shifts by $90^{\circ}$. The modified

630 eccentricity equation may prove especially helpful in future experiments when more data is

631 available or data is collected aimed at direct tests of the model so that fits can be made to

632 evaluate model parameters in a more precise way.

634 Figure A2: Demonstrates how various parameters of the modified eccentricity fitting

635 equation affect the fitting curve. A) The parameter $k$ of Eq. 7 mainly shifts the curve along

636 the abscissa without compressing the curve much. B) The parameter $m$ mainly compresses

637 the curve so that for instance the slope of the decreasing eccentricity at the end of the curve

638 increases. C) The parameter $n$ scales the curve along the ordinate. D) Given that $A_{m a g}$ is

639 fixed the parameter $B_{\text {mag }}$ determines the eccentricity and precision of the model magnetic

640 compass itself (see Eq. $1 \& 6$ ) and determines the eccentricity at $S F(\omega)_{\circ \circ l}^{*}=0$.

\section{Table and Figure Legends}


642 Table 1: Light-dependent orientation responses in non-passerine birds, amphibians, and

643 insects. $N=$ normal orientation towards an expected and meaningful direction, $D=$

644 disorientation, $S=$ shifted orientation $90^{\circ}$ in relation to the normal orientation. The

645 wavelength of the monochromatic light is shown at the top and the numbers show the

646 irradiance in $10^{15}$ quanta $/ \mathrm{m}^{2} / \mathrm{s}$. (Data taken from Freake and Phillips, 2005; Phillips and

647 Borland, 1992a; Phillips and Borland, 1992b; Phillips and Sayeed, 1993; Vacha et al.,

648 2008; Wiltschko and Wiltschko, 1998; Wiltschko et al., 2007b).

650 Table 2: Expected orientation responses predicted by the cue integration model in different

651 orientation tests with illumination by two to three different monochromatic light sources.

652 See text for details.

653

654 Figure 1: Hypothetical model compasses. A) A hypothetical magnetic compass consisting

655 of a circular array of cells. Cell spike frequency is coded by gray scale such that the darker

656 the cell the more excited it is. The directional firing pattern (see text) is represented

657 mathematically as radial plots of spike frequencies forming an ellipse (middle). B) A

658 simplified 2D hypothetical color gradient compass. The compass consists of a circular array

659 of cells receiving input from photoreceptors that sample the horizon around all $360^{\circ}$.

660 Scattering of sunlight on the atmosphere result in a skylight color gradient with a relative

661 high amount of long wavelength light in direction of the sun (light grey part of the

662 outermost circle) and a relative high amount of UV light (or shorter wavelengths in general)

663 in the anti solar direction (dark grey part of the outermost circle). In the solar and anti solar

664 direction (solar meridian) the difference in the irradiance of long wavelength and UV is 
665

666 cells aligned along the solar meridian are therefore maximally excited whereas there's a

667 minimal excitation in directions 90 relative to the solar meridian. The directional firing

668 pattern is therefore represented as an ellipse (middle) like in A. (grayscale code of compass

669 cells as in A). C) If the hypothetical compass sensory system in B is placed in

670 monochromatic light within a wavelength that specifically excites the long wavelength or

671 UV detectors only, the sensory system will perceive a large difference in long wavelength

672 and UV light in all directions all units will be highly excited (all units are dark gray). The

673 resultant directional firing pattern is thus forming a circle rather than an ellipse.

674

675 Figure 2: Model integration (solid line) between the hypothetical magnetic compass

676 (dotted line) and the hypothetical color gradient compass (dashed line). Major and minor

677 compass axes are arbitrarily set to 70 spikes/s and 35 spikes/s, and 100 spikes/s and 35

678 spikes/s for the magnetic and color gradient compass, respectively (see text). A) The

679 integration leads to a compass with a higher contrast between the North-South and East-

680 West axes. B) If, for instance, the two cues are misaligned (here by $15^{\circ}$ ) the hypothetical

681 integration compass will show a clear direction along the axis of the most prominent cue.

683 Figure 3: The outcome of the integration process in monochromatic light at different radial

684 spike frequency values, $S F(\omega)_{\text {sol }}^{*}$, of the color gradient (dashed line). Dotted line $=$

685 magnetic compass, solid line = integrated compass. A) At low input $\left(S F(\omega)_{\odot \circ l}^{\circ}=20\right)$ into

686 the color gradient compass (e.g. low intensity of the monochromatic light; see text) there is 32 
687 only a little effect and the animals are still normally oriented. B) As the input increases

$688\left(S F(\omega)_{\circ o l}^{\prime}=52\right)$ the resultant integrated compass has significant lobes along both axes

689 presumably resulting in disorientation. C) As the input further increases $\left(S F(\omega)_{\infty \infty 2}^{\prime}=7 \mathbf{5}\right)$

690 the East-West axis starts dominating and the result is a $90^{\circ}$ orientation shift of the animals.

691 D-E) The more the input into the color gradient compass increases from here

$692\left(\operatorname{SF}(\omega)_{\circ o l}^{\prime}=150\right.$ and 400), the more the contrast between the East-West and North-South

693 axis deteriorates and the animals will be expected to show disorientation again.

695 Figure 4: Categorical orientation responses as a function of light wavelength and

696 irradiance. The model predicted orientation responses are outlined together with

697 hypothetical absorption spectra (thick solid lines) of the hypothetical color gradient system

698 assumed to be consisting of UV and LW sensitive detectors. The arrows show predictions

699 of sequences of orientation responses. $N=$ normal orientation, $D=$ disorientation, and $S=$

700 shifted orientation. See text for details.

701

702 Figure 5: Model predicted changes in eccentricity (Eq. 5) of the integrated compass as the

703 circular excitation of the color gradient compass, $S E(\omega)^{\prime}$ ', increases. The higher the

704 eccentricity, the better the integrated compass points out directions. Shown are also

705 references to Fig. 3 in which the corresponding directional firing pattern of the integrated

706 compass can be seen. Solid line $=$ predicted normal orientation, dotted line $=$ predicted 
707 disorientation, and dashed line $=$ predicted shifted orientation. Calculated with $A_{\text {mag }}=70$ $708 \quad$ and $B_{\text {mag }}=35$.

710 Figure 6: The literature data on orientation responses of passerines under monochromatic

711 light of different wavelengths and irradiances. Shown is also the normalized photon catches

712 of visual cones from a representative passerine, the blue tit (Parus caeruleus), including oil

713 droplet absorption $(U V S=$ ultraviolet sensitive cones, $S W S=$ short wave sensitive cones,

$714 M W S=$ mid wave sensitive cones and $L W S=$ long wave sensitive cones). The normalized

715 absorption spectrum of rods $(R O D S)$ and the LWS pigments present in double cones $(D C$;

716 double cones generally lack oil droplets) are also shown. Shifted orientation (open

717 diamonds with a cross line) includes both observed $90^{\circ}$ shifted bimodal orientation and

718 unimodal so-called "fixed orientations" of these deviated $45^{\circ}$ or more from the normal

719 orientation. Overlapping data points are shown as a horizontal row of points and their

720 common origin shown with a cross. The numbered arrows indicate trends of orientation

721 shifts that were predicted by the model as shown in Fig. 4. Vision data for single cones was

722 taken from Hart and Hunt (2007). Double cone absorption spectrum was calculated from

723 Govardovskii et al (2000) with no oil droplet correction. Orientation data was taken from

724 (Stapput (2006) doctorial thesis data referenced in: Johnsen et al., 2007; Muheim et al.,

725 2002a; Munro et al., 1997; Rappl et al., 2000; Wiltschko et al., 2007a; Wiltschko et al.,

726 2005; Wiltschko et al., 2008; Wiltschko and Wiltschko, 1995b; Wiltschko and Wiltschko,

727 1999b; Wiltschko and Wiltschko, 2000; Wiltschko and Wiltschko, 2001; Wiltschko and

728 Wiltschko, 2002; Wiltschko and Wiltschko, 2005; Wiltschko et al., 2000; Wiltschko et al., 
729 2001; Wiltschko et al., 1993; Wiltschko et al., 2003; Wiltschko et al., 2004a; Wiltschko et 730 al., 2004b).

732 Figure 7: Model fits of compass eccentricity to literature r-values at wavelengths or

733 irradiances where adequate data were available. Statistics of the fits are summarized in each

734 figure (see methods for details). Compass eccentricity fitted to literature r-values of birds as

735 a: A) function of irradiance at a constant wavelength of $424 \mathrm{~nm}$. B) function of irradiance at

736 a constant wavelength of $565 \mathrm{~nm}$. C) function of wavelength within a constant range of

737 irradiances between $6-14 \cdot 10^{15}$ quanta $/ \mathrm{m}^{2} / \mathrm{s}$. D) function of wavelength within a constant

738 range of irradiances between $43-54 \cdot 10^{15}$ quanta $/ \mathrm{m}^{2} / \mathrm{s}$. E) Compass eccentricity fitted to

739 literature r-values of red-spotted newts as a function of wavelength at a constant

740 irradiances of $45 \cdot 10^{15}$ quanta $/ \mathrm{m}^{2} / \mathrm{s}$ (except $22 \cdot 10^{15}$ quanta $/ \mathrm{m}^{2} / \mathrm{s}$ at $400 \mathrm{~nm}$ ). F) Summary

741 of all fits to the bird data. Each data point was transferred back into the numerical domain

742 of the model by a transformation based on the values of the parameters $n, k$, and $m$ of the

743 fitting curve.

\section{References}

745 Able, K.P., 1991. Common themes and variations in animal orientation systems. Am. Zool. $746 \quad 31,157-167$.

747 Adair, R.K., 1999. Effects of very weak magnetic fields on radical pair reformation.

$748 \quad$ Bioelectromagnetics 20, 255-263.

749 Batschelet, E., 1981. Circular Statistics in Biology. Academic Press, London. 
Beason, R.C., and Nichols, J.E., 1984. Magnetic orientation and magnetically sensitive material in a transequatorial migratory bird. Nature 309, 151-153.

Bernard, G.D., and Wehner, R., 1977. Functional similarities between polarization vision and color vision. Vis. Res. 17, 1019-1028.

Cameron, D.A., and Easter, S.S., 1993. The cone photoreceptor mosaic of the green sunfish (Leomis cyanellus). Visual Neurosci. 10, 375-384.

Cintolesi, F., Ritz, T., Kay, C.W.M., Timmel, C.R., and Hore, P.J., 2003. Anisotropic recombination of an immobilized photoinduced radical pair in a 50-mu T magnetic field: a model avian photomagnetoreceptor. Chem. Phys. 294, 385-399.

Coemans, M.A.J.M., and Vos, J., On the perception of polarized light by the homing pigeon, Biological faculty, University of Utrecht 1992, pp. 1-190.

Coemans, M.A.J.M., Hzn, J.J.V., and Nuboer, J.F.W., 1994. The relationship between celestial color gradients and the position of the sun with regard to the sun compass. Vis. Res. 34, 1461-1470.

Coughlin, D.J., and Hawryshyn, C.W., 1995. A cellular basis for polarized light vision in rainbow trout. J. Comp. Physiol. A 176, 261-272.

Davila, A.F., Fleissner, G., Winklhofer, M., and Petersen, N., 2003. A new model for a magnetoreceptor in homing pigeons based on interacting clusters of superparamagnetic magnetite. Phys. Chem. Earth 28, 647-652.

Davila, A.F., Winklhofer, M., Shcherbakov, V.P., and Petersen, N., 2005. Magnetic pulse affects a putative magnetoreceptor mechanism. Biophys. J. 89, 56-63.

Deutschlander, M.E., Phillips, J.B., and Borland, S.C., 1999. The case for light-dependent magnetic orientation in animals. J. Exp. Biol. 202, 891-908. 
773 Diebel, C.E., Proksch, R., Green, C.R., Neilson, P., and Walker, M.M., 2000. Magnetite defines a vertebrate magnetoreceptor. Nature 406, 299-302.

775 Edmonds, D.T., 1992. A magnetite null detector as the migrating bird's compass. Proc.

776 Roy. Soc. Lond. B 249, 27-31.

777 Efimova, O., and Hore, P.J., 2008. Role of exchange and dipolar interactions in the radical 778 pair model of the avian magnetic compass. Biophys. J. 94, 1565-1574.

779 Flamarique, I.N., and Hawryshyn, C.W., 1998. The common white sucker (Catostomus commersoni): a fish with ultraviolet sensitivity that lacks polarization sensitivity. J.

Flamarique, I.N., and Browman, H.I., 2000. Wavelength-dependent polarization orientation in Daphnia. J. Comp. Physiol. A 186, 1073-1087. Comp. Physiol. A 182, 331-341.

Fleissner, G., Stahl, B., Thalau, P., Falkenberg, G., and Fleissner, G., 2007. A novel concept of Fe-mineral-based magnetoreception: histological and physicochemical data from the upper beak of homing pigeons. Naturwissenschaften 94, 631-642.

Fleissner, G., Holtkamp-Rotzler, E., Hanzlik, M., Winklhofer, M., Fleissner, G., Petersen, N., and Wiltschko, W., 2003. Ultrastructural analysis of a putative magnetoreceptor in the beak of homing pigeons. J. Comp. Neurol. 458, 350-360.

Freake, M.J., and Phillips, J.B., 2005. Light-dependent shift in bullfrog tadpole magnetic compass orientation: Evidence for a common magnetoreception mechanism in anuran and urodele amphibians. Ethology 111, 241-254.

Golden, R.M., 1996. Mathematical Methods for Neural Network Analysis and Design. MIT Press. 
795 Govardovskii, V.I., Fyhrquist, N., Reuter, T., Kuzmin, D.G., and Donner, K., 2000. In 796 search of the visual pigment template. Visual Neurosci. 17, 509-528.

797 Hanzlik, M., Heunemann, C., Holtkamp-Rotzler, E., Winklhofer, M., Petersen, N., and 798 Fleissner, G., 2000. Superparamagnetic magnetite in the upper beak tissue of 799 homing pigeons. Biometals 13, 325-331.

800 Hart, N.S., and Hunt, D.M., 2007. Avian visual pigments: characteristics, spectral tuning, $801 \quad$ and evolution. Am. Nat. 169, S7-S26.

802 Hawryshyn, C.W., and McFarland, W.N., 1987. Cone photoreceptor mechanisms and the 803 detection of polarized light in fish. J. Comp. Physiol. A 160, 459-465.

804 Johnsen, S., and Lohmann, K.J., 2005. The physics and neurobiology of magnetoreception. $805 \quad$ Nature Reviews Neuroscience 6, 703-712.

806 Johnsen, S., and Lohmann, K.J., 2008. Magnetoreception in animals. Phys. Today 61, 29807 35.

808 Johnsen, S., Mattern, E., and Ritz, T., 2007. Light-dependent magnetoreception: quantum catches and opponency mechanisms of possible photosensitive molecules. J. Exp.

811 Kelber, A., Thunell, C., and Arikawa, K., 2001. Polarisation-dependent colour vision in Papilio butterflies. J. Exp. Biol. 204, 2469-2480.

813 Kinoshita, M., Pfeiffer, K., and Homberg, U., 2007. Spectral properties of identified

814 polarized-light sensitive interneurons in the brain of the desert locust Schistocerca 815 gregaria. J. Exp. Biol. 210, 1350-1361.

816 Kirschvink, J.L., and Gould, J.L., 1981. Biogenic magnetite as a basis for magnetic field 817 detection in animals. Biosystems 13, 181-201. 
818

819

820

821

822

823

824

825

826

827

828

829

830

831

832

833

834

835

836

837

838

Kirschvink, J.L., and Walker, M.M., Particle-size considerations for magnetite-based magnetoreceptors, in: Kirschvink, J. L., et al., Eds.), Magnetite Biomineralization and Mangetoreception in Organisms: A New Biomagnetism, Vol. 1. Plenum Press, New York \& London 1985, pp. 243-254.

Maeda, K., Henbest, K.B., Cintolesi, F., Kuprov, I., Rodgers, C.T., Liddell, P.A., Gust, D., Timmel, C.R., and Hore, P.J., 2008. Chemical compass model of avian magnetoreception. Nature 453, 387-U38.

Mouritsen, H., 1998. Redstarts, Phoenicurus phoenicurus, can orient in a true-zero magnetic field. Anim. Behav. 55, 1311-1324.

Muheim, R., 2006. Magnetic compass calibration: a single calibration reference derived from sunset/sunrise polarized light cues from the region of sky near the horizon? J. Ornithol. 147, 50-50.

Muheim, R., Backman, J., and Akesson, S., 2002a. Magnetic compass orientation in European robins is dependent on both wavelength and intensity of light. J. Exp. Biol. 205, 3845-3856.

Muheim, R., Bäckman, J., and Åkesson, S., Magnetic compass orientation in European robins is dependent on both wavelength and intensity of light, Bird Orientation: External Cues and Ecological Factors, Lund University 2002b, pp. 162 pp.

Munro, U., Munro, J.A., Phillips, J.B., and Wiltschko, W., 1997. Effect of wavelength of light and pulse magnetisation on different magnetoreception systems in a migratory bird. Austr. J. Zool. 45, 189-198. 
Parkyn, D.C., and Hawryshyn, C.W., 1993. Polarized light sensitivity in rainbow trout (Oncorhyncus mukiss) - characterization from multiunit responses in the optic nerve. J. Comp. Physiol. A 172, 493-500.

Phillips, J.B., and Borland, S.C., 1992a. Behavioural evidence for use of a light-dependent magnetoreception mechanism by a vertebrate. Nature 359, 142-144.

Phillips, J.B., and Borland, S.C., 1992b. Wavelength specific effects of light on magnetic compass orientation of the eastern red-spotted newt Notophthalmus viridescens Ethol. Ecol. \& Evol. 4, 33-42.

Phillips, J.B., and Sayeed, O., 1993. Wavelength-dependent effects of light on magnetic compass orientation in Drosophila melanogaster J. Comp. Physiol. A 172, 303-308.

Ramsden, S.D., Anderson, L., Mussi, M., Kamermans, M., and Hawryshyn, C.W., 2008. Retinal processing and opponent mechanisms mediating ultraviolet polarization sensitivity in rainbow trout (Onchorhynchus mykiss). J. Exp. Biol. 211, 1376-1385.

Rappl, R., Wiltschko, R., Weindler, P., Berthold, P., and Wiltschko, W., 2000. Orientation behavior of Garden Warblers (Sylvia borin) under monochromatic light of various wavelengths. Auk 117, 256-260.

Ritz, T., Adem, S., and Schulten, K., 2000. A model for photoreceptor-based magnetoreception in birds. Biophys. J. 78, 707-718.

Rodgers, C.T., and Hore, P.J., 2009. Chemical magnetoreception in birds: The radical pair mechanism. PNAS 106, 353-360.

Rossel, S., and Wehner, R., 1984. Celestial orientation in bees - the use of spectral cues. J. Comp. Physiol. A 155, 605-613. 
861 Sancar, A., 1999. Cryptochromes as circadian photoreceptors in man and mouse.

862 Photochem. Photobiol. 69, 5S-5S.

863 Sancar, A., 2003. Structure and function of DNA photolyase and cryptochrome blue-light

864 photoreceptors. Chem. Rev. 103, 2203-2237.

865 Schulten, K., Magnetic field effects in chemistry and biology, Festkörperprobleme XXII,

866 1982, pp. 61-83.

867 Schulten, K., Windemuth, A., and Maret, G., Model for a physiological magnetic compass,

868 in: Boccara, N., (Ed.), Biophysical Effects of Steady Magnetic Fields, Springer-

869 Verlag Berlin, Heidelberg, New York, London, Paris, Tokyo 1986, pp. 99-106.

870 Shcherbakov, V.P., and Winklhofer, M., 1999. The osmotic magnetometer: a new model

871 for magnetite-based magnetoreceptors in animals. Eur. Biophys. J. Biophy. 28, 380-

$872 \quad 392$.

873 Solov'yov, I.A., and Greiner, W., 2007. Theoretical analysis of an iron mineral-based

874 magnetoreceptor model in birds. Biophys. J. 93, 1493-1509.

875 Solov'yov, I.A., and Greiner, W., 2008. Iron-mineral-based magnetoreceptor in birds:

876 polarity or inclination compass? European Physical Journal D 51, 161-172.

877 Solov'yov, I.A., and Schulten, K., 2009. Magnetoreception through Cryptochrome May

878 Involve Superoxide. Biophys. J. 96, 4804-4813.

879 Solov'yov, I.A., Chandler, D.E., and Schulten, K., 2007. Magnetic field effects in

880 Arabidopsis thaliana cryptochrome-1. Biophys. J. 92, 2711-2726.

881 Vacha, M., Puzova, T., and Drstkova, D., 2008. Effect of light wavelength spectrum on 882 magnetic compass orientation in Tenebrio molitor. J. Comp. Physiol. A 194, 853$883 \quad 859$. 
884 Walker, M.M., 2008. A model for encoding of magnetic field intensity by magnetite-based magnetoreceptor cells. J. Theor. Biol. 250, 85-91.

Walker, M.M., Diebel, C.E., Haugh, C.V., Pankhurst, P.M., Montgomery, J.C., and Green, C.R., 1997. Structure and function of the vertbrate magnetic sense. Nature 390, 371376.

Wang, K., Mattern, E., and Ritz, T., 2006. On the use of magnets to disrupt the physiological compass of birds. Phys. Biol. 3, 220-231.

891 Weaver, J.C., Vaughan, T.E., and Astumian, R.D., 2000. Biological sensing of small field differences by magnetically sensitive chemical reactions. Nature 405, 707-709.

Wehner, R., The perception of polarised light, in: Cosens, D. J. and Vince-Prue, D., Eds.), The Biology of Photo Reception, Cambridge University Press, Cambridge 1983.

Wehner, R., The ant's celestial compass system: spectral and polarization channels, in: Lehrer, M., (Ed.), Orientation and Communication in Arthropods, Birkhäuser, Basel 1997, pp. 145-185.

Wehner, R., Bernard, G.D., and Geiger, E., 1975. Twisted and non-twisted rhabdoms and

Williams, M.N., and Wild, J.M., 2001. Trigeminally innervated iron-containing structures in the beak of homing pigeons, and other birds. Brain Research 889, 243-246.

903 Wiltschko, R., and Wiltschko, W., 1995a. Magnetic orientation in animals. Springer, $904 \quad$ Berlin.

905 Wiltschko, R., and Wiltschko, W., 1998. Pigeon homing: Effect of various wavelengths of 906 light during displacement. Naturwissenschaften 85, 164-167. 
907 Wiltschko, R., and Wiltschko, W., 1999a. The orientation system of birds - I. Compass 908 mechanisms. J. Ornithol. 140, 1-40.

909 Wiltschko, R., and Wiltschko, W., 2006. Magnetoreception. Bioessays 28, 157-168.

910 Wiltschko, R., Wiltschko, W., Davies, M.N.O., and Green, P.R., Avian orientation:

911 Multiple sensory cues and the advantage of redundancy, Perception and Motor

912 Control in Birds, Springer-Verlag, Berlin Heidelberg 1994, pp. 95-119.

913 Wiltschko, R., Stapput, K., Bischof, H.J., and Wiltschko, W., 2007a. Light-dependent

914 magnetoreception in birds: increasing intensity of monochromatic light changes the

915 nature of the response. Front. Zool. 4, doi:10.1186/1742-9994-4-5.

916 Wiltschko, R., Ritz, T., Stapput, K., Thalau, P., and Wiltschko, W., 2005. Two different

917 types of light-dependent responses to magnetic fields in birds. Curr. Biol. 15, 1518-

$918 \quad 1523$.

919 Wiltschko, R., Munro, U., Ford, H., Stapput, K., and Wiltschko, W., 2008. Light-dependent 920 magnetoreception: orientation behaviour of migratory birds under dim red light. J. $921 \quad$ Exp. Biol. 211, 3344-3350.

922 Wiltschko, W., The influence of magnetic total intensity and inclination on directions 923 preferred by migrating European Robins (Erithacus rubecula), in: Galler, S. R., et 924 al., Eds.), Animal Orientation and Navigation, NASA SP, Washington D.C. 1972, $925 \quad$ pp. $569-578$.

926 Wiltschko, W., and Wiltschko, R., 1993. Navigation in birds and other animals. J. Nav. 46, $927 \quad 174-191$. 
928 Wiltschko, W., and Wiltschko, R., 1995b. Migratory orientation of European robins is 929 affected by the wavelength as well as by a magnetic pulse. J. Comp. Physiol. A 177, $930 \quad 363-369$.

931 Wiltschko, W., and Wiltschko, R., 1999b. The effect of yellow and blue light on magnetic compass orientation in European robins, Erithacus rubecula J. Comp. Physiol. A

Wiltschko, W., and Wiltschko, R., 2000. Light-dependent magnetoreception in birds: Does directional information change with light intensity? Naturwissenschaften 87, 36-40.

Wiltschko, W., and Wiltschko, R., 2001. Light-dependent magnetoreception in birds: the behaviour of European robins, Erithacus rubecula, under monochromatic light of various wavelengths and intensities. J. Exp. Biol. 204, 3295-3302.

Wiltschko, W., and Wiltschko, R., 2002. Magnetic compass orientation in birds and its physiological basis. Naturwissenschaften $89,445-452$.

941 Wiltschko, W., and Wiltschko, R., 2005. Magnetic orientation and magnetoreception in birds and other animals. J. Comp. Physiol. A 191, 675-693.

943 Wiltschko, W., Wiltschko, R., and Munro, U., 2000. Light dependent magnetoreception in birds: the effect of intensity of 565-nm green light. Naturwissenschaften 87, 366-

946 Wiltschko, W., Gesson, M., and Wiltschko, R., 2001. Magnetic compass orientation of European robins under 565 nm green light. Naturwissenschaften 88, 387-390.

948 Wiltschko, W., Munro, U., Ford, H., and Wiltschko, R., 1993. Red light disrupts magnetic 949 orientation of migratory birds. Nature $364,525-527$. 
950

951

952

953

954

955

956

957

958

959

960

961

962

963

964

965

966

967

968

969

970

971

972

Wiltschko, W., Munro, U., Ford, H., and Wiltschko, R., 2003. Magnetic orientation in birds: non-compass responses under monochromatic light of increased intensity. Proc. Roy. Soc. Lond. B 270, 2133-2140.

Wiltschko, W., Gesson, M., Stapput, K., and Wiltschko, R., 2004a. Light-dependent magnetoreception in birds: interaction of at least two different receptors. Naturwissenschaften 91, 130-134.

Wiltschko, W., Moller, A., Gesson, M., Noll, C., and Wiltschko, R., 2004b. Lightdependent magnetoreception in birds: analysis of the behaviour under red light after pre-exposure to red light. J. Exp. Biol. 207, 1193-1202.

Wiltschko, W., Freire, R., Munro, U., Ritz, T., Rogers, L., Thalau, P., and Wiltschko, R., 2007b. The magnetic compass of domestic chickens, Gallus gallus. J. Exp. Biol. 210, 2300-2310.

Winklhofer, M., Holtkamp-Rotzler, E., Hanzlik, M., Fleissner, G., and Petersen, N., 2001. Clusters of superparamagnetic magnetite particles in the upper-beak skin of homing pigeons: evidence of a magnetoreceptor? Eur. J. Mineral. 13, 659-669.

Yorke, E.D., 1979. A possible magnetic transducer in birds. J. Theor. Biol. 77, 101-105.

Yorke, E.D., 1981. Sensivity of pigeons to small magnetic field variations. J. Theor. Biol. $89,533-537$.

Yorke, E.D., Kirschvink, J.L., Jones, D.S., and MacFadden, B.J., Energetics and sensivity considerations of ferromagnetic magnetoreceptors, in: Stehli, F. G., (Ed.), Magnetite Biomineralization and Mangetoreception in Organisms: A New Biomagnetism, Vol. 1. Plenum Press, New York \& London 1985, pp. 233-242.

Zar, J.H., 1996. Biostatistical analysis. Prentice-Hall, Inc., Upper Saddle River, N.J. USA. 45 
멈
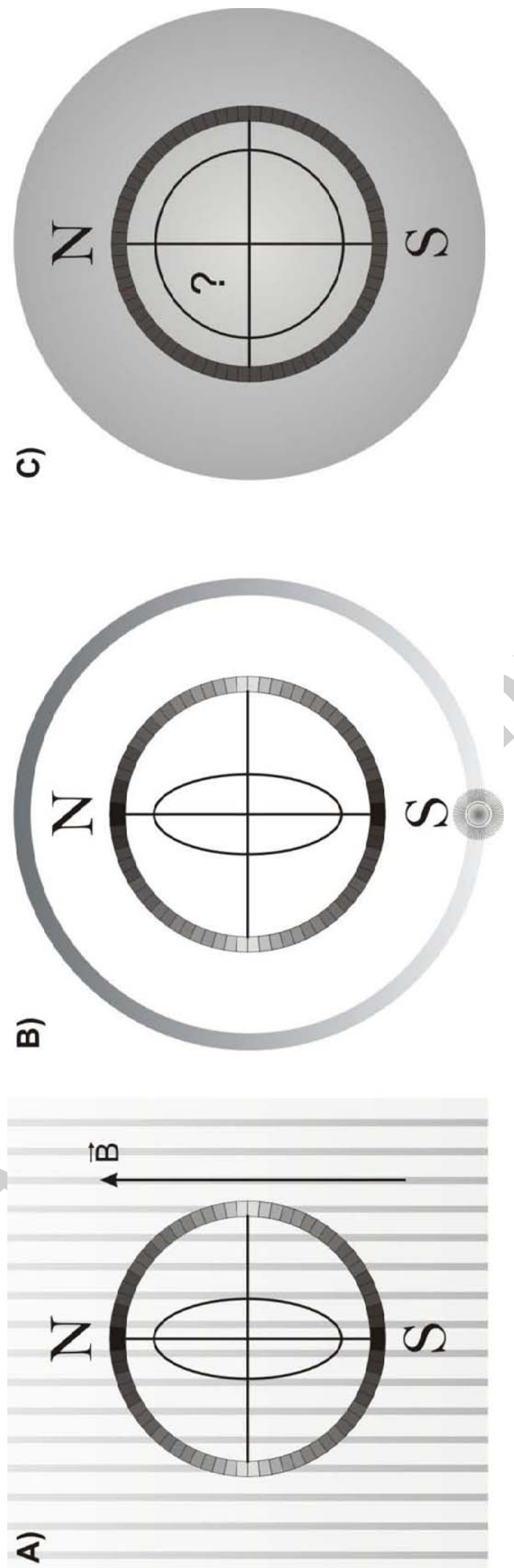


\section{FIGURE 2}
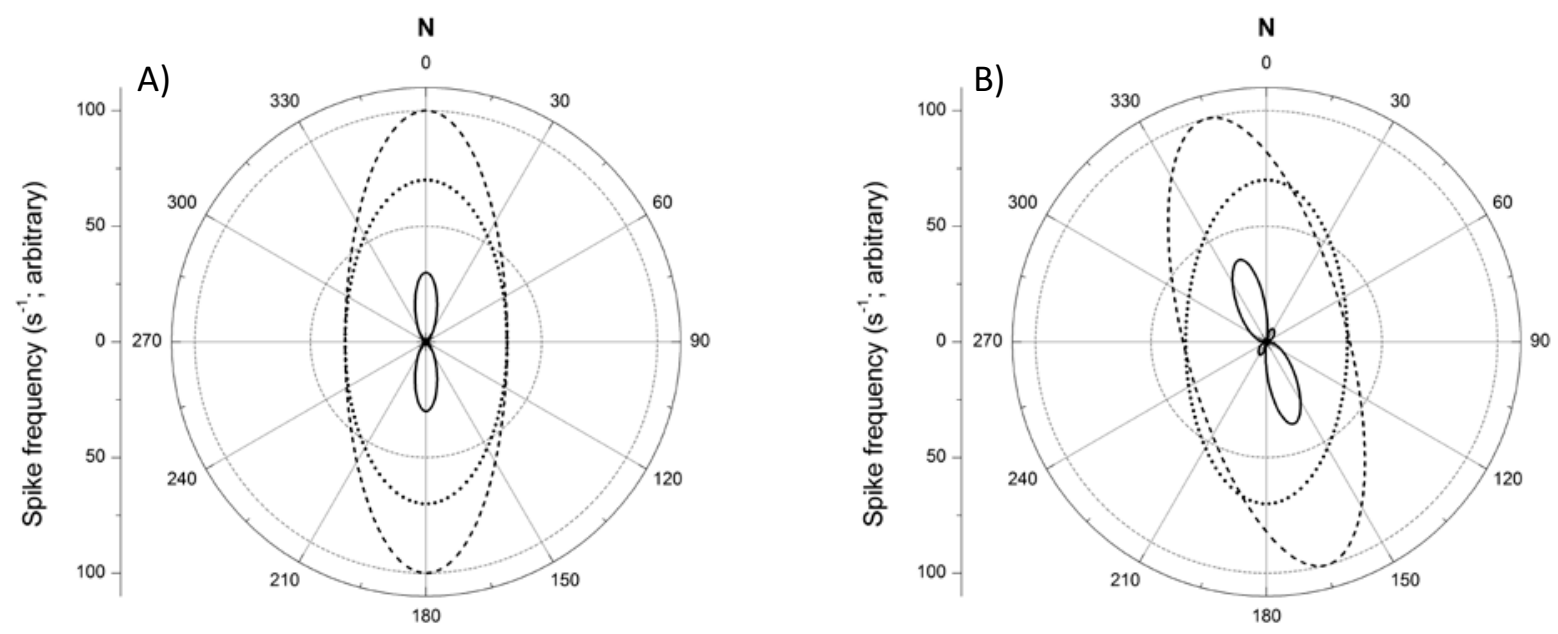


\section{FIGURE 3}
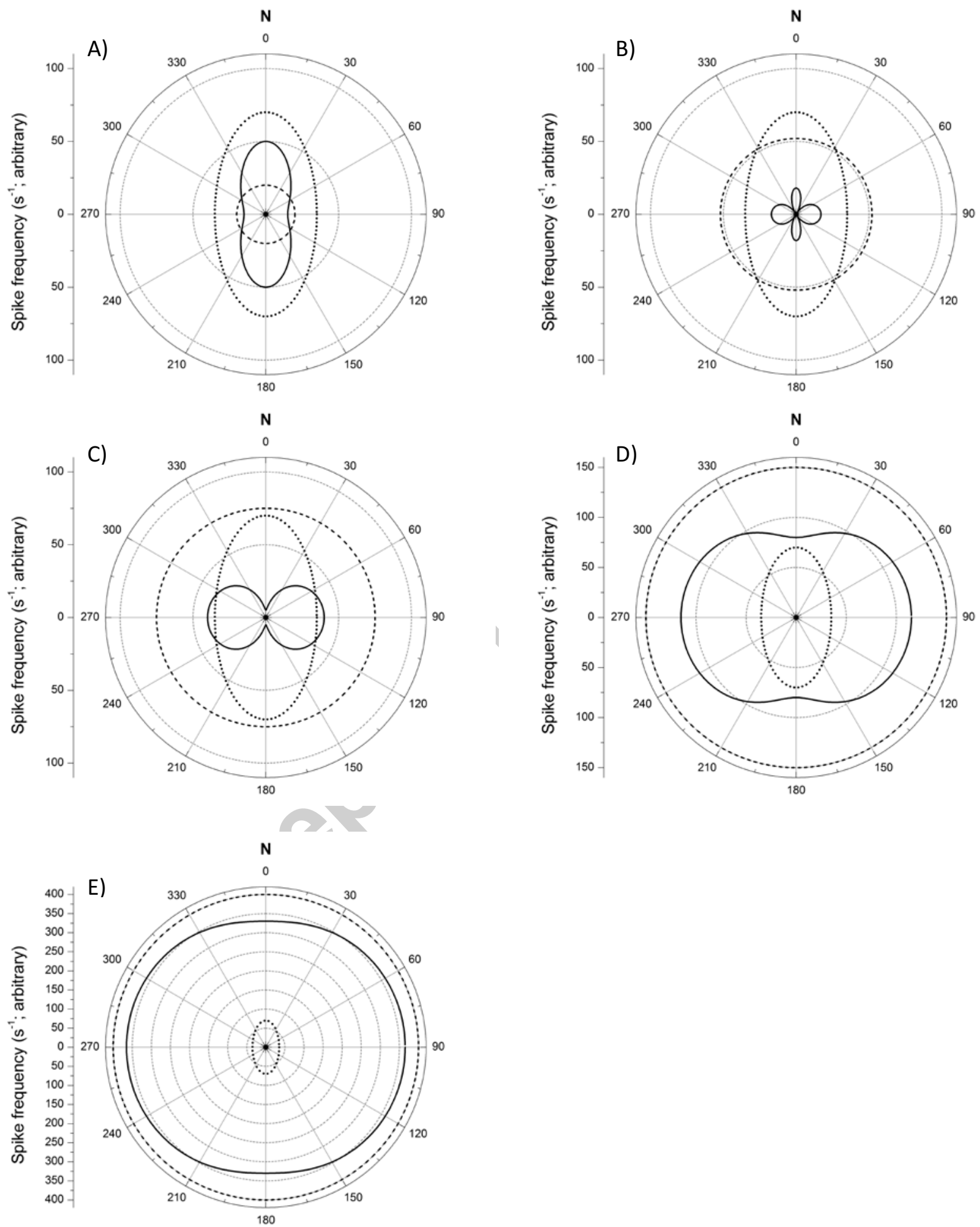
FIGURE 4

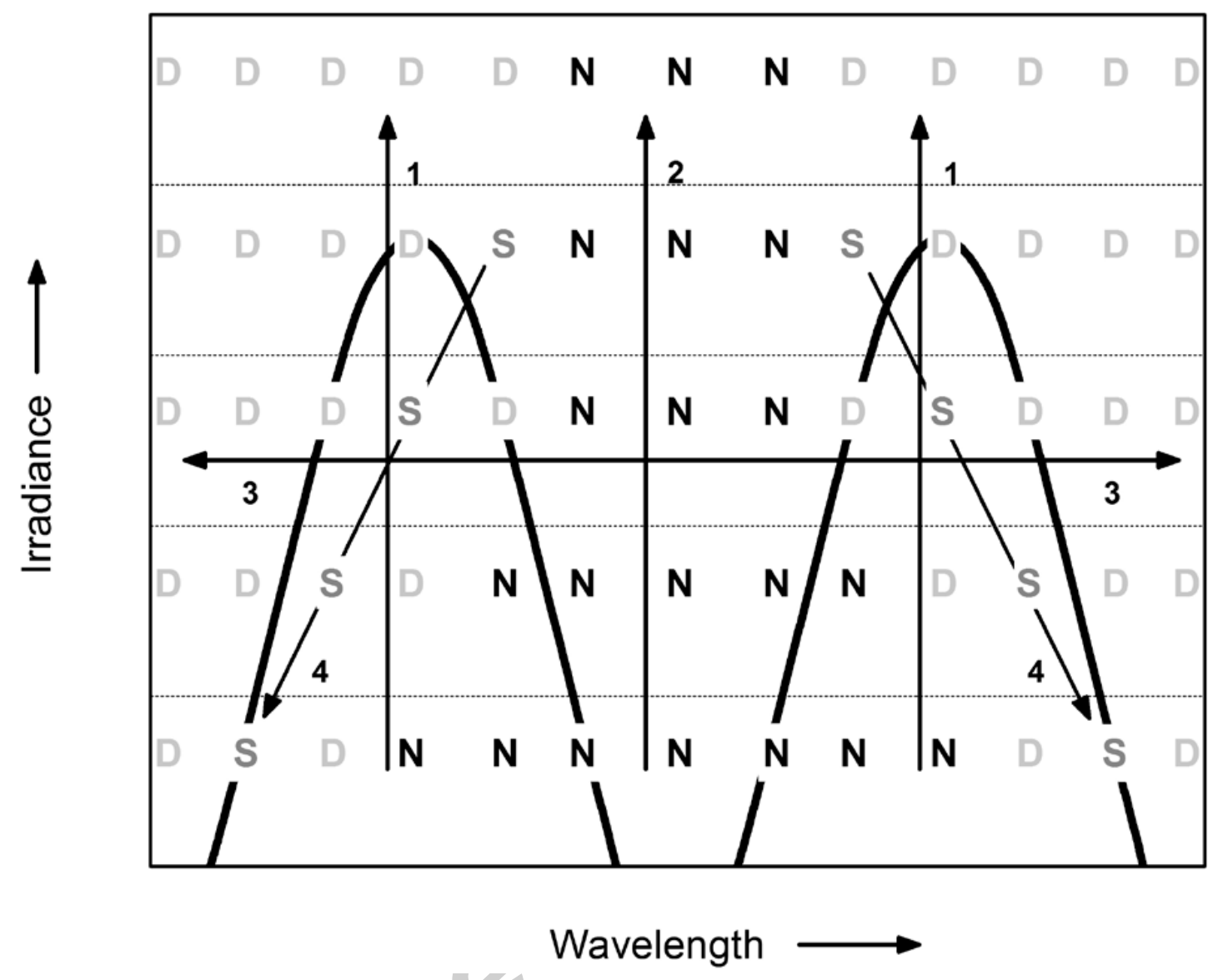


FIGURE 5

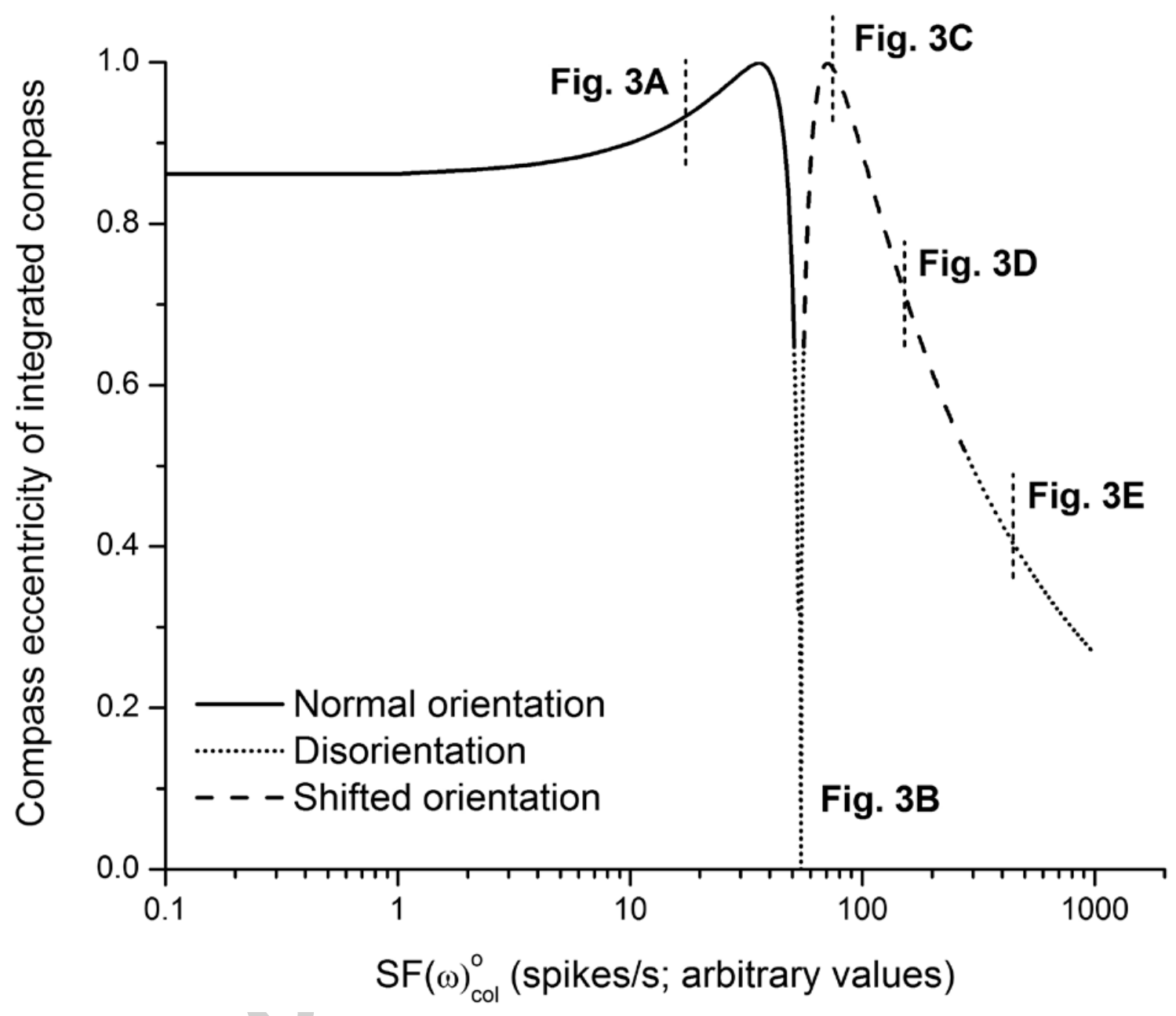


FIGURE 6

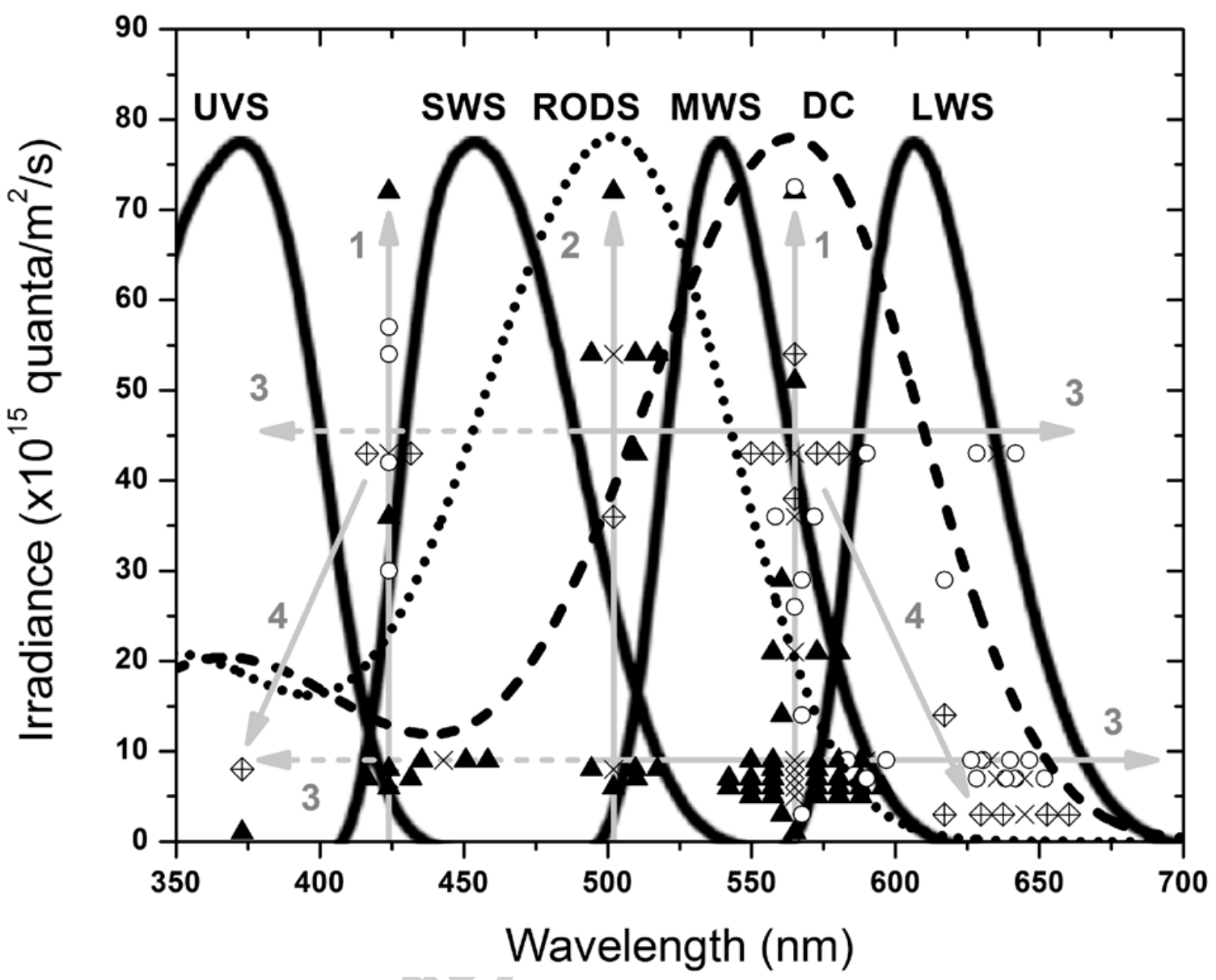


FIGURE 7

FIGURE A1

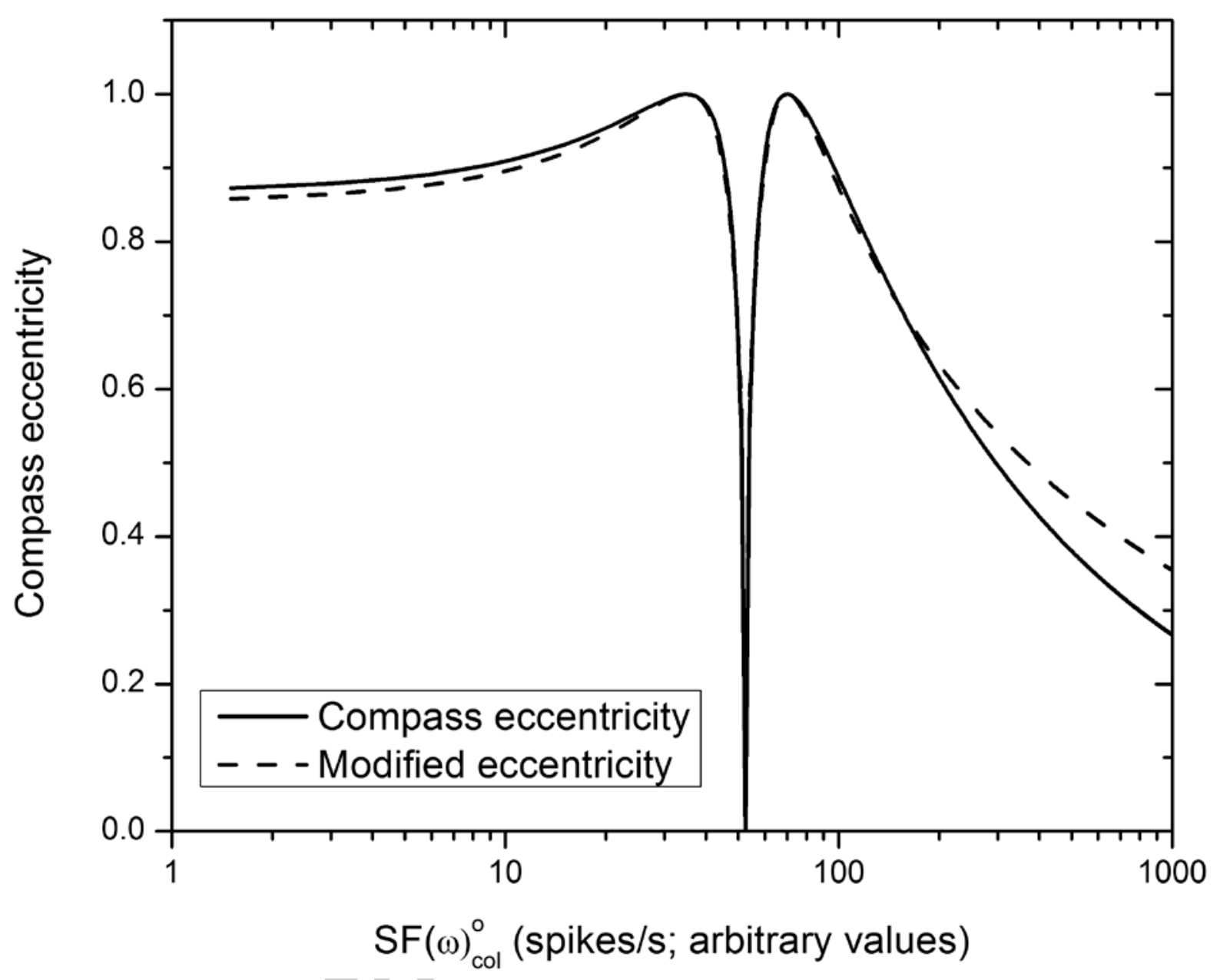


FIGURE A2

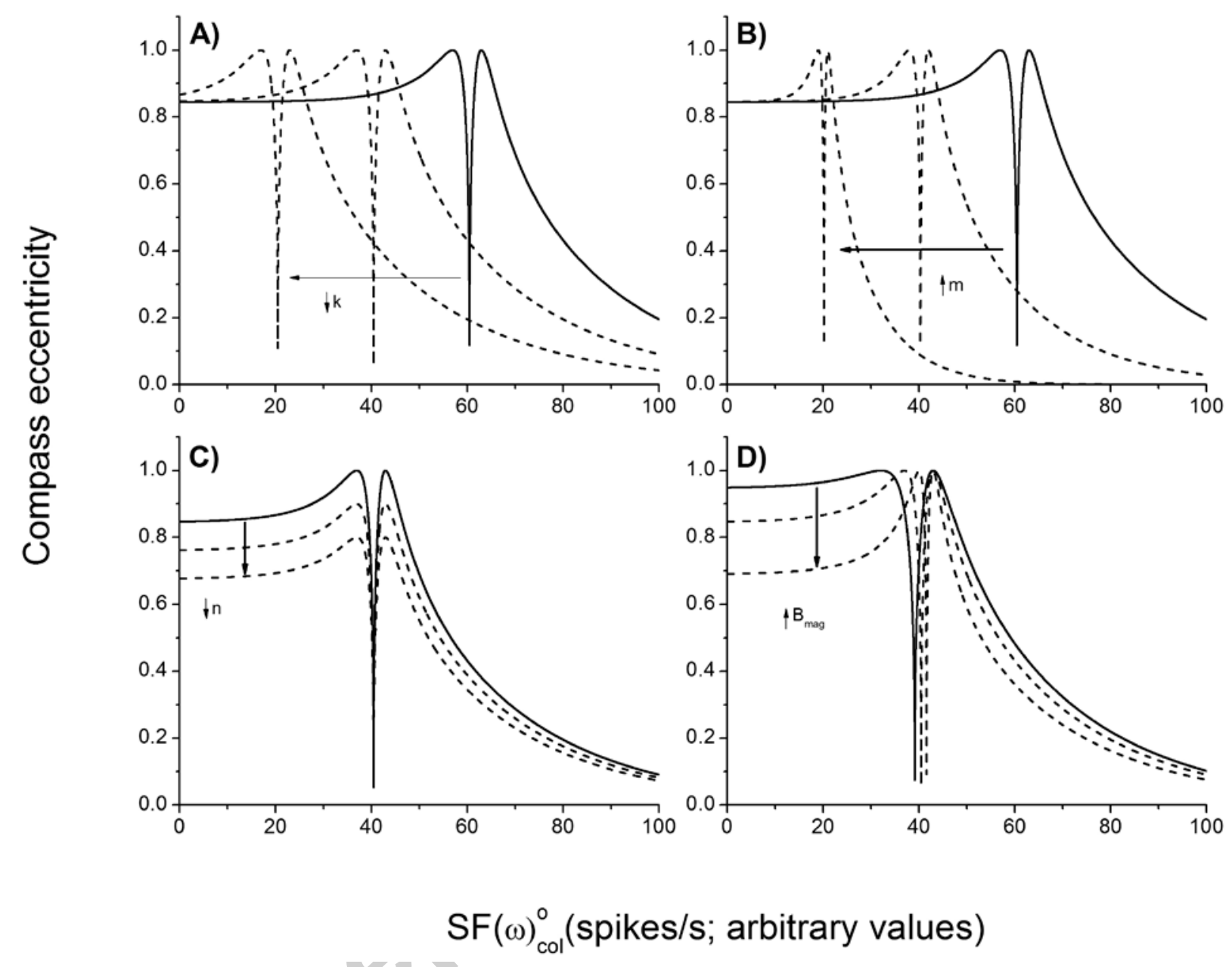




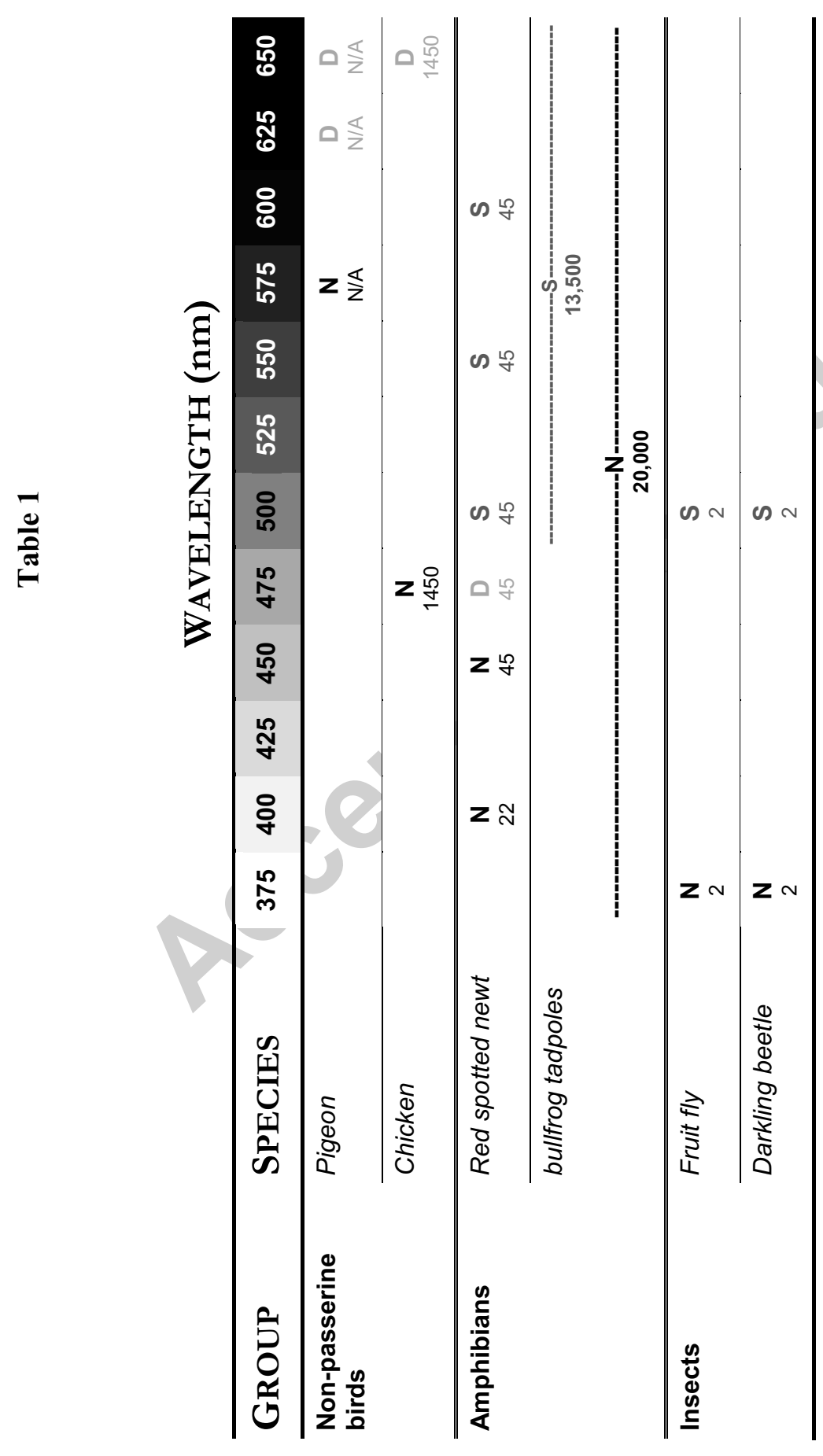




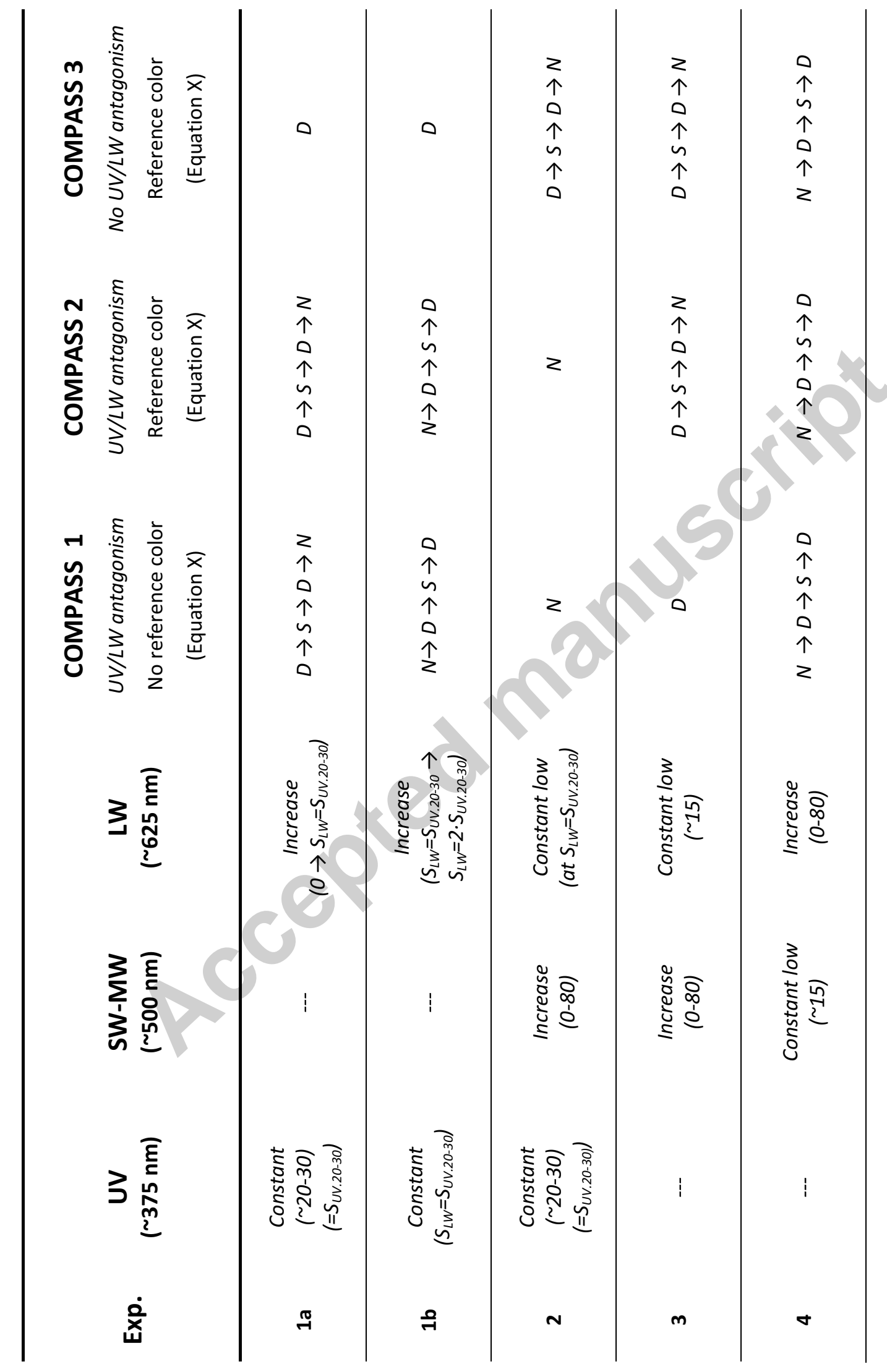

